\title{
Vibrations of a Slightly Curved Microbeam Resting on an Elastic Foundation with Nonideal Boundary Conditions
}

\author{
Gözde Sari ${ }^{1,2}$ and Mehmet Pakdemirli' ${ }^{1,2}$ \\ ${ }^{1}$ Applied Mathematics and Computation Center, Celal Bayar University, Muradiye, 45140 Manisa, Turkey \\ ${ }^{2}$ Department of Mechanical Engineering, Celal Bayar University, Muradiye, 45140 Manisa, Turkey
}

Correspondence should be addressed to Gözde Sarl; gozde.deger@cbu.edu.tr

Received 18 January 2013; Accepted 7 May 2013

Academic Editor: Mohammad Younis

Copyright (c) 2013 G. Sarı and M. Pakdemirli. This is an open access article distributed under the Creative Commons Attribution License, which permits unrestricted use, distribution, and reproduction in any medium, provided the original work is properly cited.

\begin{abstract}
An investigation into the dynamic behavior of a slightly curved resonant microbeam having nonideal boundary conditions is presented. The model accounts for midplane stretching, an applied axial load, and a small AC harmonic force. The ends of the curved microbeam are on immovable simple supports and the microbeam is resting on a nonlinear elastic foundation. The forced vibration response of curved microbeam due to the small AC load is obtained analytically by means of direct application of the method of multiple scales (a perturbation method). The effects of the nonlinear elastic foundation as well as the effect of curvature on the vibrations of the microbeam are examined. It is found that the effect of curvature is of softening type. For sufficiently high values of the coefficients, the elastic foundation and the axial load may suppress the softening behavior resulting in hardening behavior of the nonlinearity. The frequencies and mode shapes obtained are compared with the ideal boundary conditions case and the differences between them are contrasted on frequency-response curves. The frequency response and nonlinear frequency curves obtained may provide a reference for the choice of reasonable resonant conditions, design, and industrial applications of such systems. Results may be beneficial for future experimental and theoretical works on MEMS.
\end{abstract}

\section{Introduction}

Electrically actuated microbeams are mostly used in microelectromechanical systems. They have superior features such as compact size, high resolution, high sensitivity, digital output, and low-power consumption. These sensors become an attractive alternative to conventional piezoresistive sensors due to these superior features. An axial strain is applied to a microbeam to cause variations in its natural frequencies. These variations of frequency enable the microbeam to be used as a sensor to measure physical quantities such as temperature, pressure, force, and acceleration. Vibrations of electrically actuated straight microbeams in resonant sensors are of interest to many researches [1-17]. Younis and Nayfeh [1] presented response of a resonant microbeam to an electric actuation. They used a nonlinear model to account for the midplane stretching, a DC electrostatic force, and an AC harmonic force. The effect of the design parameters on the dynamic responses is discussed. Zhang and Meng [2] presented a simplified model to study the resonant responses and nonlinear dynamics of idealized electrostatically actuated microcantilever-based devices in microelectromechanical systems (MEMS). They discussed the effects of different applied voltages, the cubic nonlinear spring, and the squeeze film damping on the nonlinear and chaotic behaviors of the system. Mestrom et al. [3] modeled the dynamics of a MEMS resonator that potentially captures the observed behavior. Apart from the model consisting of a mechanical and an electrical (measurement) part, the effect of thermal noise was also estimated. With the proposed model, a quantitative match between the simulation and experimental results was established such that a good starting point is achieved for a more thorough modeling procedure. Jia et al. [4] presented an analytical study on the forced vibration of electrically actuated microswitches near resonance region. They used the perturbation based method of averaging to solve the governing nonlinear partial-differential equation. Abu-Salih and Elata [5] analyzed the electromechanical buckling of 
a prestressed layer bonded to an elastic foundation. The effect of stiffening and softening elastic foundations on the postbuckling behavior of the system is discussed. Rivlin and Elata [6] proposed a method for designing nonlinear elastic springs with increasing stiffness to counteract the nonlinear effects of electrostatic attraction. They intended to increase the dynamic range of the parallel-plates electrostatic actuator and force a linear relation between the applied voltage and the displacement in their study. They achieved a good agreement between experiments and modal prediction for concept of nonlinear springs. Kong et al. [7] solved the dynamic problems of Bernoulli-Euler beams analytically on the basis of modified couple stress theory. The size effect on the microbeam's natural frequencies for two kinds of boundary conditions that were simply supported microbeam and cantilever microbeam was investigated. It was found that the natural frequencies of the microbeams predicted by the new model were larger than that predicted by the classical beam model.

In recent years, curved microbeams have been considered in the microelectromechanical systems (MEMS) because of their superior features than the straight microbeams such as their bistability nature and performance in large strokes. The curved microbeams can be resting in either of two states and do not need energy to keep the mechanism in either of their bistable states. They have a practical use in applications such as micro-valves, electrical micro-relays, microswitches and micro-filters thanks to their snap-through action. This action is static phenomenon due to static forces. They may exhibit pull-in instability due to an interaction between mechanical and electrostatic nonlinearities, when the curved microbeams are actuated by electro static forces. It is very important that the critical voltage which causes pull-in instability is defined because of the microbeams' failure over the critical voltages [8].

Casals-Terre and Shkel [9] investigated theoretically and experimentally the use of mechanical resonance to switch between states of a bistable nature. Qiu et al. [10] analyzed a bistable mechanism that plays a vital role in the development of MEMS mechanisms. Modal analysis and finite element analysis simulation of the curved beam were used to predict and design its bistable behavior. Zhang et al. [11] studied theoretically and experimentally the snap-through and the pull-in instabilities of the micromachined arch-shaped beams under an electrostatic loading. Their analysis was static and showed that the effect of arch configuration was important for the snap-through instability.

Krylov et al. [12] presented results of theoretical and experimental investigation of initially curved microbeams by electrostatic force. Results of their work provided a better understanding of the physical phenomena of such systems. Good agreement was observed among their theoretical and experimental results. Das and Batra [13] studied transient analysis of the curved microbeam. They emphasized that the microarches had advantages as MEMS electrodes because the curved microbeams could have a larger operational range without the pull-in instability than a corresponding straight microbeam. Younis et al. [14] presented an analytic approach and reduced order model to investigate electrically actuated microbeam. Results of their work showed that the pull-in voltage corresponds to a saddle-node bifurcation.

Ouakad and Younis [8] studied the dynamic behavior of clamped-clamped micromachined arches. They calculated the natural frequencies and mode shapes of the arch for various values of DC voltages and initial rises. Their results showed softening type behavior for the resonance frequency for all DC and AC loads as well as the initial rise of the arch.

An electrically actuated imperfect microbeam has been investigated very recently $[15,16]$. Ruzziconi et al. [15] studied the nonlinear response of an electrically actuated microbeam which had imperfections due to microfabrications. Their theoretical and experimental results were in good agreement.

Ruzziconi et al. [16] also developed a dynamical integrity analysis to interpret and predict the experimental response of the microbeam which had imperfections. The integrity charts provide invaluable information for engineering design of such structures.

The nonlinear vibrations of a slightly curved macroscale beam have been investigated in the literature $[18,19]$. Öz et al. [18] investigated nonlinear vibrations of the slightly curved beams which were resting on a nonlinear elastic foundation. The amplitude and phase modulation equations were derived for the case of primary resonances. Effects of the nonlinear elastic foundation and curvature on the vibrations of the microbeam were examined. It is found that the effect of the curvature is of softening type. The elastic foundation may suppress the softening behavior resulting in a hardening behavior of the nonlinearity. Öz and Pakdemirli [19] studied two-to-one internal resonances between any two modes of vibration of shallow curved beams. They discussed the steady-state solutions and their stability.

Nonideal boundary condition concept was proposed recently [17, 20-23]. Deviations from ideal conditions were formulated using perturbation theory. A nonideal simple support may have small deflections or small moments or a combination of both. Similarly nonideal built-in support may have small deflections and small slopes.

Nonideal boundary conditions of both macroscale and micro-scale beams have been investigated recently. Pakdemirli and Boyaci [20-23] applied the concept of nonideal boundary conditions to the macroscale beam problem. The boundaries were assumed to allow small deflections. They showed that the small variations of the deflections at the ends may affect the frequencies of the response. Ekici and Boyaci [17] investigated the effect of nonideal boundary conditions on the vibrations of straight microbeams. They showed that the nonideal boundary conditions could cause shifting of the frequencies or the frequency-response curves to the left or right side or no shifting, depending on the mode numbers, axial forces, deflections, and moments on the boundaries.

In this study, the nonlinear model of the microbeam accounts for the slightly curved beam with an AC electrostatic force, midplane stretching, and an applied axial load. The microbeam is bonded to an elastic foundation with cubic nonlinearities. The equations of motion are made nondimensional and solved by the method of multiple scales, a perturbation technique. Approximate response of the microbeam 


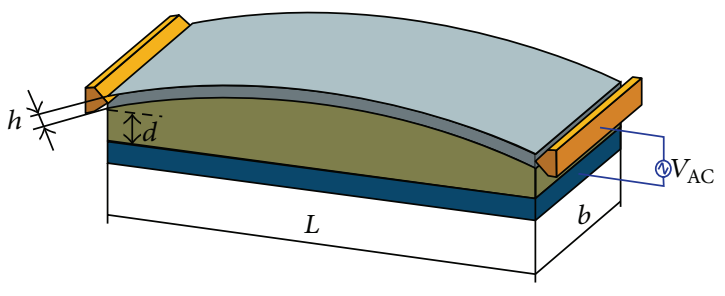

FIGURE 1: A simply supported slightly curved microbeam resting on a nonlinear elastic foundation.

to a primary-resonance excitation is obtained. Effects of the elastic foundation, the dielectric constants, the axial load, the AC component of the voltage, the curvature, and the strength of the midplane stretching on the vibrations of the microbeam are analyzed. The frequencies and mode shapes obtained are compared with the ideal boundary conditions case and the deviations from the ideal case are shown on the frequency-response curves. This investigation provides an understanding of the nonlinear dynamic characteristics of slightly curved microbeams having nonideal boundary conditions.

\section{Equation of Motion}

A schematic view of a slightly curved microbeam bonded to an elastic nonlinear foundation is presented in Figure 1. The kinetic and potential energies of the system are

$$
\begin{gathered}
T=\frac{1}{2} \int_{0}^{1} \rho A \dot{w}^{* 2} d x^{*}, \\
V=\frac{1}{2} \frac{E A}{1-v^{2}} \int_{0}^{1}\left(u^{* \prime}+Z_{0}^{* \prime} w^{* \prime}+\frac{1}{2} w^{* \prime}\right)^{2} d x^{*} \\
+\frac{1}{2} \frac{E I}{1-\nu^{2}} \int_{0}^{1} w^{* \prime \prime 2} d x^{*}+\frac{1}{2} N^{*} \int_{0}^{1} w^{* \prime 2} d x^{*} \\
+\int_{0}^{1}\left(\frac{1}{2} k_{1} w^{* 2}+\frac{1}{4} k_{2} w^{* 4}\right) d x^{*},
\end{gathered}
$$

where $w^{*}$ is the transverse displacement, $u^{*}$ is the longitudinal displacement, $Z_{0}^{*}$ is the arbitrary initial rise function (curvature), $E$ is the modulus of elasticity, $N^{*}$ is the axial load, $A$ is the rectangular cross-sectional area $(A=b h), v$ is the poisson's ratio, and $h$ and $b$ are the microbeam thickness and width, respectively. $d$ is the gap width, $I$ is the area moment of inertia with respect to the neutral axis $\left(I=(1 / 12) b h^{3}\right)$, $\rho$ is the volumetric density, $k_{1}$ is the linear spring constant for the elastic foundation, and $k_{2}$ is the nonlinear spring constant for the elastic foundation. $x^{*}$ and $t^{*}$ are the spatial and time variables, respectively, and prime and dot denote differentiation with respect to these variables. The first term is the energy due to the stretching of the microbeam, the second term is the energy due to the bending of the microbeam, the third term is the energy due to the tensile or compressive axial load, and the last term is the energy due to the elastic foundation.
The Hamilton Principle can be employed to obtain the equation of motion for transverse vibrations of the microbeam. The final result is

$$
\begin{gathered}
\rho A \frac{\partial^{2} w^{*}}{\partial t^{* 2}}+\frac{E I}{1-v^{2}} \frac{\partial^{4} w^{*}}{\partial x^{* 4}}+2 \mu^{*} \frac{\partial w^{*}}{\partial t^{*}}+k_{1} w^{*}+k_{2} w^{* 3} \\
=\left[\frac{E A}{L\left(1-v^{2}\right)}\left(\frac{\partial^{2} Z_{0}^{*}}{\partial x^{* 2}}+\frac{\partial^{2} w^{*}}{\partial x^{* 2}}\right)\right] \\
\quad \times\left[\int_{0}^{1}\left(\frac{1}{2}\left(\frac{\partial w^{*}}{\partial x^{*}}\right)^{2}+\frac{\partial Z_{0}^{*}}{\partial x^{*}} \frac{\partial w^{*}}{\partial x^{*}}\right) d x^{*}\right] \\
+N^{*} \frac{\partial^{2} w^{*}}{\partial x^{* 2}}+\frac{\varepsilon_{0} b\left(V_{\mathrm{AC}} \cos \left(\Omega^{*} t^{*}\right)\right)^{2}}{2\left(d+Z_{0}+w\right)^{2}},
\end{gathered}
$$

where $\mu^{*}$ is the viscous damping and $\varepsilon_{0}$ is the dielectric constant of the vacuum. The last term in the right hand side of (2) represents the parallel plate electric forces [24] assuming a complete overlapping area between the microbeam and the stationary electrode. Here it is assumed that the microbeam is simply supported from both ends. However, it is assumed that the boundary condition at the right hand side is not ideal and some slight variations occur in the right hand side deflections

$$
\begin{gathered}
w^{*}\left(0, t^{*}\right)=0, \quad \frac{\partial^{2} w^{*}\left(0, t^{*}\right)}{\partial x^{* 2}}=0, \\
w^{*}\left(L, t^{*}\right)=\alpha\left(t^{*}\right), \quad \frac{\partial^{2} w^{*}\left(L, t^{*}\right)}{\partial x^{* 2}}=0,
\end{gathered}
$$

where $\varepsilon$ is a small perturbation parameter denoting that the variations in deflections are small.

Introducing the dimensionless variables for universality of the results:

$$
w=\frac{w^{*}}{d}, \quad x=\frac{x^{*}}{L}, \quad t=\frac{t^{*}}{T}, \quad Z_{0}=\frac{Z_{0}{ }^{*}}{d},
$$

the equation of motion and the boundary conditions become

$$
\begin{gathered}
\frac{\partial^{4} w}{\partial x^{4}}+\frac{\partial^{2} w}{\partial t^{2}}+2 \mu \frac{\partial w}{\partial t}+\alpha_{1} w+\alpha_{2} w^{3} \\
=\Gamma\left(\frac{d^{2} Z_{0}}{d x^{2}}+\frac{\partial^{2} w}{\partial x^{2}}\right)\left[\int_{0}^{1}\left(\frac{1}{2}\left(\frac{\partial w}{\partial x}\right)^{2}+\frac{d Z_{0}}{d x} \frac{d w}{d x}\right) d x\right] \\
+N \frac{\partial^{2} w}{\partial x^{2}}+\alpha_{3} F_{e} \\
w(0, t)=0, \quad \frac{\partial^{2} w(0, t)}{\partial x^{2}}=0 \\
w(1, t)=\varepsilon^{3} \alpha(t), \quad \frac{\partial^{2} w\left(1, t^{*}\right)}{\partial x^{2}}=0
\end{gathered}
$$

where

$$
F_{e}=\frac{V_{A C}^{2} \cos ^{2} \Omega t}{\left(1+Z_{0}+w\right)^{2}}
$$


TABLE 1: The nondimensional parameters.

\begin{tabular}{lc}
\hline $\begin{array}{l}\text { Parameter } \\
\alpha_{1}=\frac{k_{1} L^{4}\left(1-v^{2}\right)}{A E d^{2}}\end{array}$ & Definition \\
$\alpha_{2}=k_{2} L^{4}\left(\frac{1-v^{2}}{E A}\right)$ & The linear coefficient of the foundation \\
$\alpha_{3}=\frac{\varepsilon_{0} b\left(1-v^{2}\right) L^{4}}{2 d^{3} E I}$ & The electric force parameter \\
$\Gamma=\frac{12 d^{2}}{h^{2}}$ & The midplane stretching parameter \\
$\mu=\frac{\mu^{*} L^{2}}{\sqrt{\rho A E I}}$ & The damping parameter \\
$N=\frac{N^{*} L^{2}\left(1-v^{2}\right)}{E I}$ & The axial force parameter
\end{tabular}

The model is a nondimensional integropartial-differential equation with nonlinear terms and $T$ is a time scale which is chosen as

$$
T=\ell^{2} \sqrt{\frac{\rho A\left(1-v^{2}\right)}{E I}} .
$$

The dimensionless parameters are defined in Table 1.

\section{Perturbation Analysis}

We investigate the nonlinear vibrations of a simply supported slightly curved microbeam subject to a small AC electric load. We analyzed its nonlinear response to a primary-resonance excitation because it is the case that is mostly encountered in resonator applications.

The direct-perturbation method is applied to the integropartial-differential equation. This direct treatment has some advantages over the more common method of discretizing the partial-differential system and then applying perturbations [25-28]. Solutions are assumed to be of the form:

$$
\begin{aligned}
w(x, t ; \varepsilon)= & \varepsilon w_{1}\left(x, T_{0}, T_{1}, T_{2}\right)+\varepsilon^{2} w_{2}\left(x, T_{0}, T_{1}, T_{2}\right) \\
& +\varepsilon^{3} w_{2}\left(x, T_{0}, T_{1}, T_{2}\right)
\end{aligned}
$$

where $T_{0}=t$ is the usual fast time scale and $T_{1}=\varepsilon t$ and $T_{2}=\varepsilon^{2} t$ are the slow time scales. Time derivatives are defined as

$$
\begin{gathered}
\frac{d}{d t}=D_{0}+\varepsilon D_{1}+\varepsilon^{2} D_{2}, \\
\frac{d^{2}}{d t^{2}}=D_{0}^{2}+2 \varepsilon D_{0} D_{1}+\varepsilon^{2}\left(D_{1}^{2}+2 D_{0} D_{2}\right),
\end{gathered}
$$

where $D_{n}=\partial / \partial T_{n}$.

In order that the nonlinearity balances the effects of voltage excitation, $\alpha_{3}$ is rescaled as $\varepsilon^{3} \alpha_{3}$. The perturbation technique is limited to small AC amplitudes. Hence, the results of this study are valid under effects of small AC load and slight curvature. The dynamic pull-in [8] phenomenon was not investigated in this study.

If (9) and (10) are substituted into (5), the following equations are obtained at each order of $\varepsilon$ :

Order $\varepsilon$

$$
\begin{gathered}
w_{1}^{\imath v}+D_{0}^{2} w_{1}+\alpha_{1} w_{1}-N w_{1}^{\prime \prime}-\Gamma Z_{0}^{\prime \prime} \int_{0}^{1} Z_{0}^{\prime} w_{1}^{\prime} d x=0, \\
w_{1}\left(0, T_{0}, T_{1}, T_{2}\right)=0, \quad w_{1}^{\prime \prime}\left(0, T_{0}, T_{1}, T_{2}\right)=0, \\
w_{1}\left(1, T_{0}, T_{1}, T_{2}\right)=0, \quad w_{1}^{\prime \prime}\left(1, T_{0}, T_{1}, T_{2}\right)=0,
\end{gathered}
$$

$\operatorname{Order} \varepsilon^{2}$

$$
\begin{aligned}
& w_{2}^{\imath v}+D_{0}^{2} w_{2}+\alpha_{1} w_{2}-N w_{2}^{\prime \prime}-\Gamma Z_{0}^{\prime \prime} \int_{0}^{1} Z_{0}^{\prime} w_{2}^{\prime} d x \\
& =\frac{1}{2} \Gamma Z_{0}^{\prime \prime} \int_{0}^{1} w_{1}^{\prime 2} d x+\Gamma w_{1}^{\prime \prime} \int_{0}^{1} Z_{0}^{\prime} w_{1}^{\prime} d x-2 D_{0} D_{1} w_{1}, \\
& w_{2}\left(0, T_{0}, T_{1}, T_{2}\right)=0, \quad w_{2}^{\prime \prime}\left(0, T_{0}, T_{1}, T_{2}\right)=0 \\
& w_{2}\left(1, T_{0}, T_{1}, T_{2}\right)=0, \quad w_{2}^{\prime \prime}\left(1, T_{0}, T_{1}, T_{2}\right)=0
\end{aligned}
$$

$\operatorname{Order} \varepsilon^{3}$

$$
\begin{aligned}
& w_{3}^{\imath v}+ D_{0}^{2} w_{3}+\alpha_{1} w_{3}-\Gamma Z_{0}^{\prime \prime} \int_{0}^{1} Z_{0}^{\prime} w_{3}^{\prime} d x-N w_{3}^{\prime \prime} \\
&=-2 D_{0} D_{1} w_{2}-\left(D_{1}^{2}+2 D_{0} D_{2}\right) w_{1}-2 \mu D_{0} w_{1} \\
&-\alpha_{2} w_{1}^{3}+\Gamma Z_{0}^{\prime \prime} \int_{0}^{1} w_{1}^{\prime} w_{2}^{\prime} d x \\
&+\frac{1}{2} \Gamma w_{1}^{\prime \prime} \int_{0}^{1} w_{1}^{\prime 2} d x+\Gamma w_{1}^{\prime \prime} \int_{0}^{1} Z_{0}^{\prime} w_{2}^{\prime} d x \\
&+\Gamma w_{2}^{\prime \prime} \int_{0}^{1} Z_{0}^{\prime} w_{1}^{\prime} d x+\frac{\alpha_{3} V_{\mathrm{AC}}^{2} \cos ^{2} \Omega t}{\left(1+Z_{0}\right)^{2}} \\
& w_{3}\left(0, T_{0}, T_{1}, T_{2}\right)=0, \quad w_{3}^{\prime \prime}\left(0, T_{0}, T_{1}, T_{2}\right)=0 \\
& w_{3}\left(1, T_{0}, T_{1}, T_{2}\right)=\alpha\left(T_{0}, T_{1}, T_{2}\right) \\
& w_{3}^{\prime \prime}\left(1, T_{0}, T_{1}, T_{2}\right)=0 .
\end{aligned}
$$

At $\operatorname{order} \varepsilon$, the solution may be expressed as

$$
w_{1}\left(x, T_{0}, T_{1}, T_{3}\right)=\left(A\left(T_{1}, T_{2}\right) e^{i \omega T_{0}}+c c\right) Y(x),
$$

where $c c$ stands for the complex conjugates of the preceding terms. The mode shapes satisfy the following differential system:

$$
\begin{aligned}
& Y^{I V}-\beta^{2} Y-\Gamma Z_{0}^{\prime \prime} \int_{0}^{1} Z_{0}^{\prime} Y^{\prime} d x-N Y^{\prime \prime}=0, \\
& Y(0)=Y^{\prime \prime}(0)=Y^{\prime \prime}(1)=0, \quad Y(1)=0,
\end{aligned}
$$

where

$$
\beta^{2}=\omega^{2}-\alpha_{1}
$$




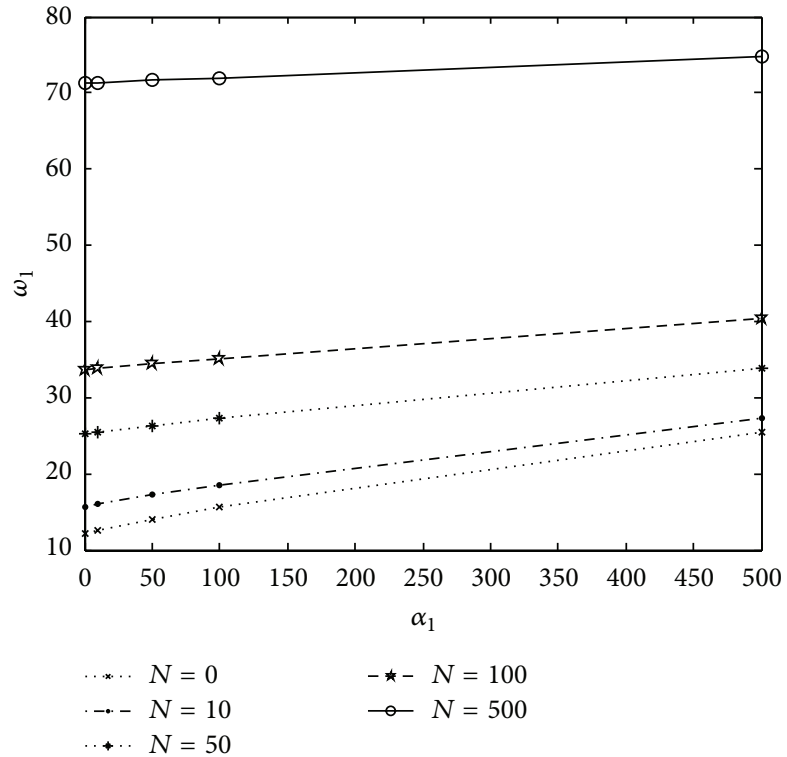

(a)

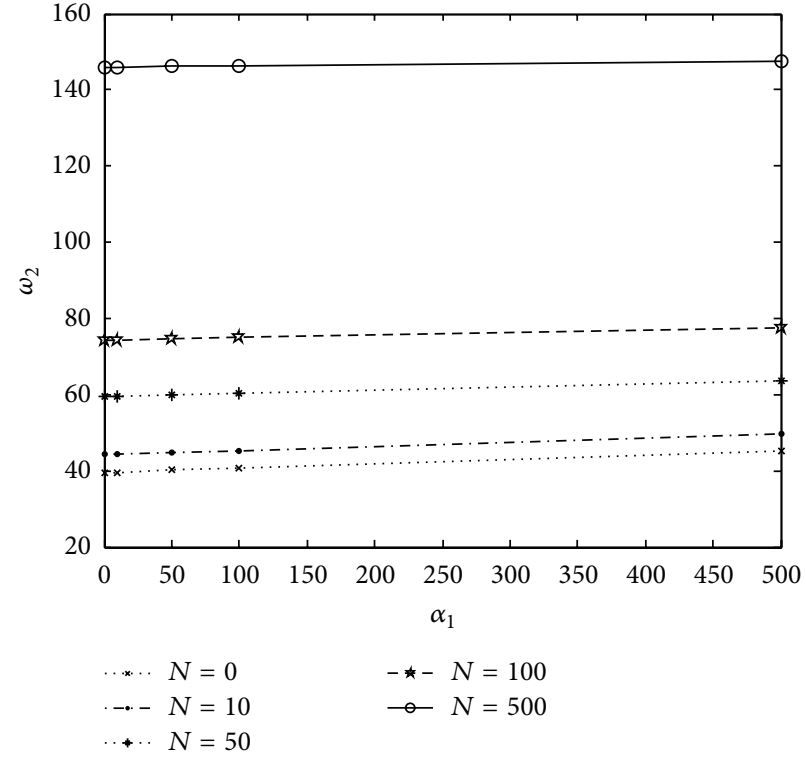

(b)

Figure 2: The first two natural frequencies versus linear coefficients of the elastic foundation for various axial force parameters.

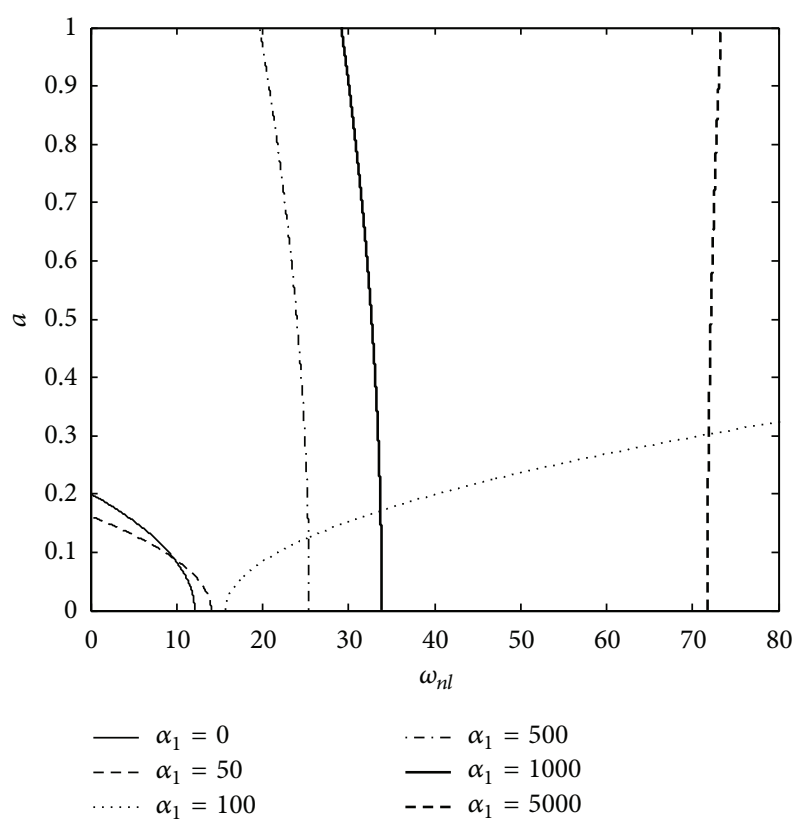

FIGURE 3: Nonlinear frequency versus amplitude for the first mode: $\varepsilon=1, \alpha_{2}=10, N=0, \Gamma=15$, and $B_{1}=0$.

Defining

$$
B=\Gamma \int_{0}^{1} Z_{0}^{\prime} Y^{\prime} d x
$$

one has

$$
Y^{I V}-\beta^{2} Y-B Z_{0}^{\prime \prime}-N Y^{\prime \prime}=0
$$

By choosing a sinusoidal curvature function

$$
Z_{0}=\sin \pi x
$$

solutions can be obtained for two different cases. If $B=0$, the solutions are

$$
Y=C \sin n \pi x, \quad \beta=\sqrt{n^{4} \pi^{4}+n^{2} \pi^{2} N} \quad n=2,3,4, \ldots .
$$

If $B \neq 0$, then the solution is

$$
Y=C \sin \pi x, \quad \beta=\sqrt{\frac{3}{2} \pi^{4}+N \pi^{2}} \quad n=1 .
$$

From the solvability condition at order $\varepsilon^{2}$ (see details of finding solvability conditions in [29]), one obtains

$$
D_{1} A=0, \quad A=A\left(T_{2}\right) .
$$

A solution can be written at this order of the form:

$$
w_{2}=\left(A^{2} e^{2 i \omega T_{0}}+c c\right) \phi_{1}(x)+2 A \bar{A} \phi_{2}(x) .
$$

If one normalizes the eigenfunctions at order $\varepsilon$ by requiring $\int_{0}^{1} Y^{2} d x=1$, one has

$$
Y(x)=\sqrt{2} \sin n \pi x .
$$

Substituting (23) into (12) yields

$$
\begin{gathered}
\phi_{1}^{I V}-\left(4 \omega^{2}-\alpha_{1}\right) \phi_{1}-N \phi_{1}^{\prime \prime}-\Gamma Z_{0}^{\prime \prime} \int_{0}^{1} Z_{0}^{\prime} \phi_{1}^{\prime} d x \\
=\frac{1}{2} \Gamma Z_{0}^{\prime \prime} \int_{0}^{1} Y^{\prime 2} d x+\Gamma Y^{\prime \prime} \int_{0}^{1} Z_{0}^{\prime} Y^{\prime} d x, \\
\phi_{2}^{I V}-N \phi_{2}^{\prime \prime}+\alpha_{1} \phi_{2}-\Gamma Z_{0}^{\prime \prime} \int_{0}^{1} Z_{0}^{\prime} \phi_{2}^{\prime} d x
\end{gathered}
$$



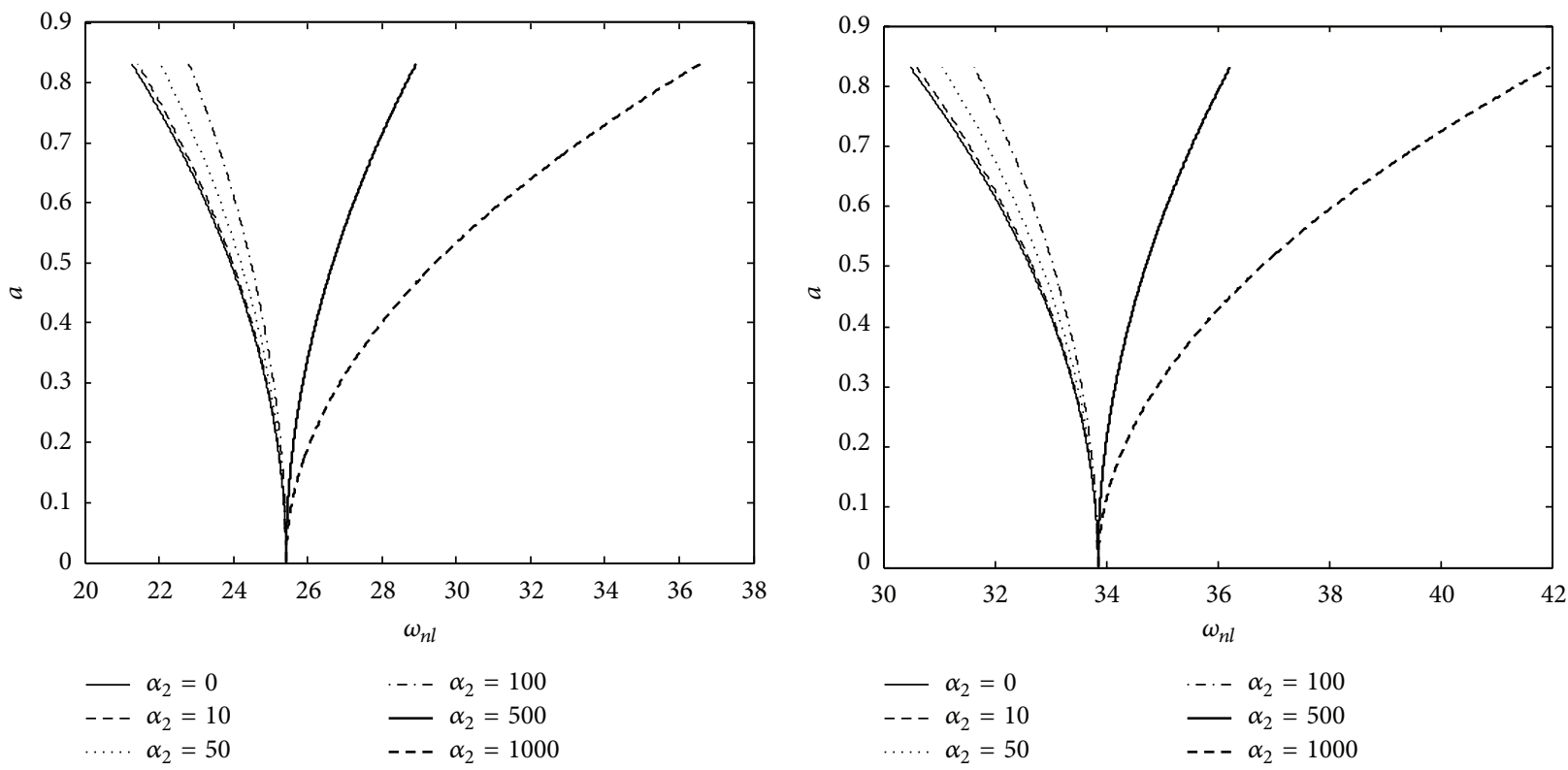

(b)

FIGURE 4: Nonlinear frequency versus amplitude for the first mode: (a) $\varepsilon=1, \alpha_{1}=500, N=0, \Gamma=15$, and $B_{1}=0$; (b) $\varepsilon=1, \alpha_{1}=$ $1000, N=0, \Gamma=15$, and $B_{1}=0$.

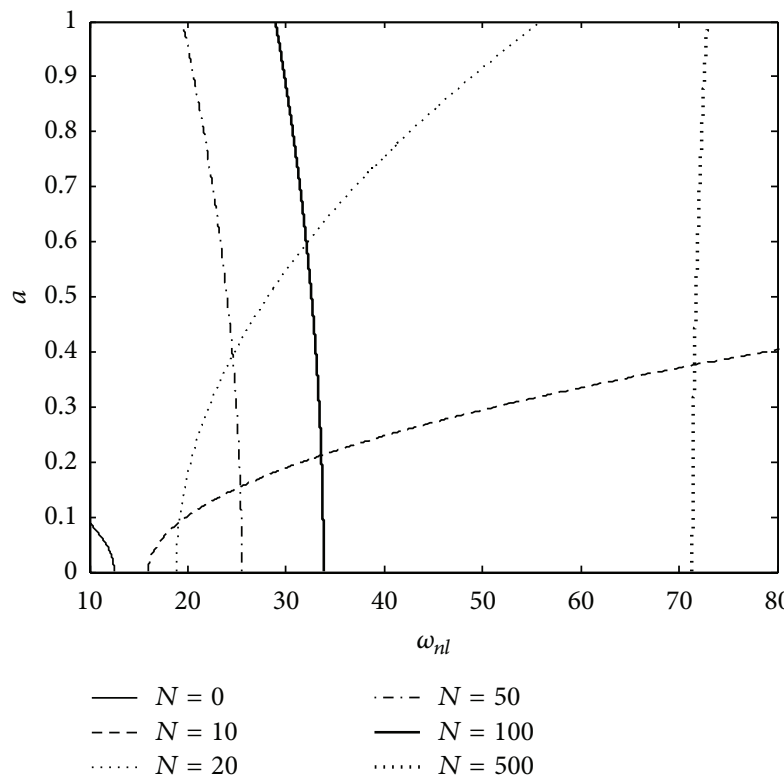

FIGURE 5: Nonlinear frequency versus amplitude for the first mode: $\varepsilon=1, \alpha_{1}=10, \alpha_{2}=0, \Gamma=15$, and $B_{1}=0$.

$$
\begin{gathered}
=\frac{1}{2} \Gamma Z_{0}^{\prime \prime} \int_{0}^{1} Y^{\prime 2} d x+\Gamma Y^{\prime \prime} \int_{0}^{1} Z_{0}^{\prime} Y^{\prime} d x, \\
\phi_{i}(0)=0, \quad \phi_{i}(1)=0, \quad \phi_{i}^{\prime \prime}(0)=0, \\
\phi_{i}^{\prime \prime}(1)=0, \quad i=1,2 .
\end{gathered}
$$

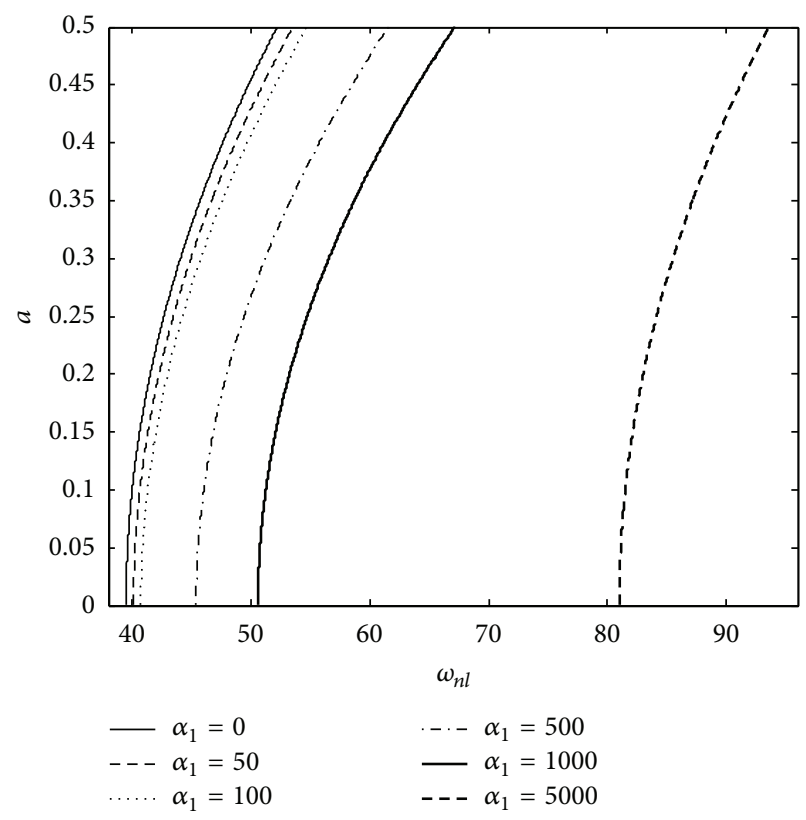

Figure 6: Nonlinear frequency versus amplitude for the second mode: $\varepsilon=1, \alpha_{2}=10, N=0, \Gamma=15$, and $B_{1}=0$.

For $B=0(n \neq 1)$ case the mode shapes at this order are

$$
\begin{aligned}
\phi_{1}= & \frac{\Gamma n^{2} \pi^{4}}{8 n^{4} \pi^{4}+8 n^{2} \pi^{2} N+6 \alpha_{1}-2 N \pi^{2}-2 \pi^{4}-\Gamma \pi^{4}} \\
& \times \sin \pi x, \\
\phi_{2}= & -\frac{\Gamma n^{2} \pi^{4}}{2 \pi^{4}+2 N \pi^{2}+2 \alpha_{1}+\Gamma \pi^{4}} \sin \pi x \quad n=2,3, \ldots
\end{aligned}
$$




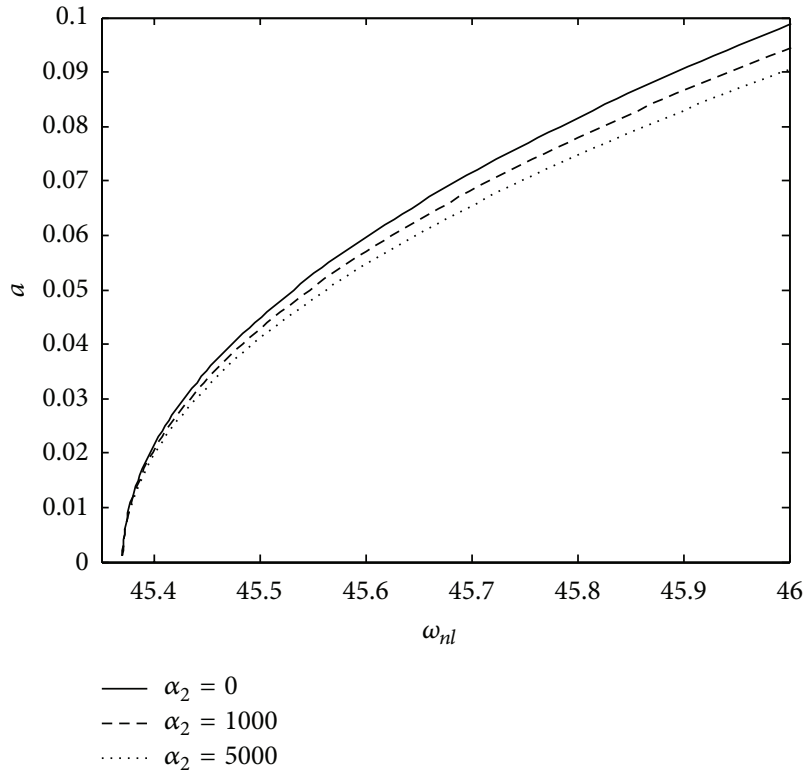

FIGURE 7: Nonlinear frequency versus amplitude for the second mode: $\varepsilon=1, \alpha_{1}=500, N=0, \Gamma=15$, and $B_{1}=0$.

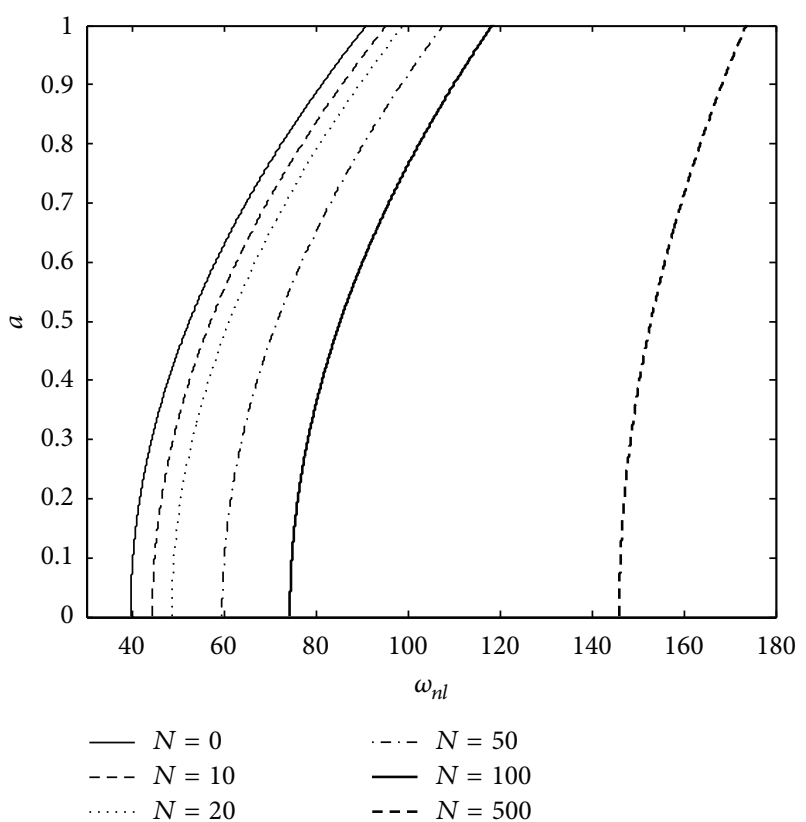

FIGURE 8: Nonlinear frequency versus amplitude for the second mode: $\varepsilon=1, \alpha_{1}=10, \alpha_{2}=0, \Gamma=15$, and $B_{1}=0$.

and for $B \neq 0(n=1)$ case

$$
\begin{gathered}
\phi_{1}=\frac{3 \Gamma \pi^{4}}{10 \pi^{4}+6 N \pi^{2}+6 \alpha_{1}-\Gamma \pi^{4}} \sin \pi x, \\
\phi_{2}=-\frac{3 \Gamma \pi^{4}}{2 \pi^{4}+2 N \pi^{2}+2 \alpha_{1}+\Gamma \pi^{4}} \sin \pi x \quad n=1 .
\end{gathered}
$$

The solution at order $\varepsilon^{3}$ is written as

$$
w_{3}\left(x, T_{0}, T_{2}\right)=\varphi\left(x, T_{2}\right) e^{i \omega T_{0}}+W\left(x, T_{0}, T_{2}\right)+c c .
$$

The excitation frequency is taken as

$$
2 \Omega=\omega+\varepsilon^{2} \sigma,
$$

where $\sigma$ is a detuning parameter of $O(\varepsilon), \varphi$ is the part of solution related to the secular terms, and $W$ is the part of solution related to the nonsecular terms. Inserting expressions (14), (23), (28), and (29) into (13) and considering only the terms producing secularities, one has

$$
\begin{gathered}
\varphi^{i v}-\left(\omega^{2}-\alpha_{1}\right) \varphi-\Gamma Z_{0}^{\prime \prime} \int_{0}^{1} Z_{0}^{\prime} \varphi^{\prime} d x-N \varphi^{\prime \prime} \\
=-2 i \omega Y\left(D_{2} A+\mu A\right) \\
+A^{2} \bar{A}\left(-3 \alpha_{2} Y^{3}+\Gamma Y^{\prime \prime} b_{4}+2 \Gamma Y^{\prime \prime} b_{5}\right. \\
+\Gamma b_{7}\left(\phi_{1}^{\prime \prime}+2 \phi_{2}^{\prime \prime}\right)+\frac{3}{2} \Gamma n^{2} \pi^{2} Y^{\prime \prime} \\
\left.+\Gamma b_{2} Z_{0}^{\prime \prime}+2 \Gamma b_{3} Z_{0}^{\prime \prime}\right)+\frac{\alpha_{3} b_{6} V_{\mathrm{AC}}^{2} e^{i \sigma T_{2}}}{4}, \\
\varphi(0)=0, \quad \varphi(1)=B_{1} A\left(T_{2}\right), \\
\varphi^{\prime \prime}(0)=0, \quad \varphi^{\prime \prime}(1)=0,
\end{gathered}
$$

where

$$
\begin{gathered}
b_{1}=\int_{0}^{1} Y^{4} d x, \quad b_{2}=\int_{0}^{1} Y^{\prime} \phi_{1}^{\prime} d x, \\
b_{3}=\int_{0}^{1} Y^{\prime} \phi_{2}^{\prime} d x, \quad b_{4}=\int_{0}^{1} Z_{0} \phi_{1}^{\prime} d x, \\
b_{5}=\int_{0}^{1} Z_{0}^{\prime} \phi_{2}^{\prime} d x, \quad b_{6}=\int_{0}^{1} \frac{Y}{\left(1+Z_{0}\right)^{2}} d x, \\
b_{7}=\int_{0}^{1} Y^{\prime} Z_{0}^{\prime} d x, \quad \int_{0}^{1} Y^{2} d x=1 .
\end{gathered}
$$

Here $B_{1}$ is a constant representing the magnitude of the deflection of the right end of the microbeam.

The homogenous problem of (15) possesses a nontrivial solution. For the non-homogenous problem of (30) to possess a solution, a solvability condition should be satisfied (see [29] for details of calculating this condition). For the present problem, the solvability condition requires

$$
M A=-2 i \omega\left(D_{2} A+\mu A\right)-\lambda A^{2} \bar{A}+\frac{\alpha_{3}}{4} V_{\mathrm{AC}}^{2} b_{6} e^{i \sigma T_{2}},
$$

where

$$
\begin{gathered}
\lambda=-\int_{0}^{1} Y\left[-3 \alpha_{2} Y^{3}+\Gamma Y^{\prime \prime} b_{4}+2 \Gamma b_{5} Y^{\prime \prime}\right. \\
+\Gamma b_{7}\left(\phi_{1}^{\prime \prime}+2 \phi_{2}^{\prime \prime}\right)+\frac{3}{2} \Gamma n^{2} \pi^{2} Y^{\prime \prime} \\
\left.+\Gamma b_{2} Z_{0}^{\prime \prime}+2 \Gamma b_{3} Z_{0}^{\prime \prime}\right] d x \\
M=B_{1} \sqrt{2} \pi n\left(n^{2} \pi^{2}+N\right) \cos \pi n .
\end{gathered}
$$




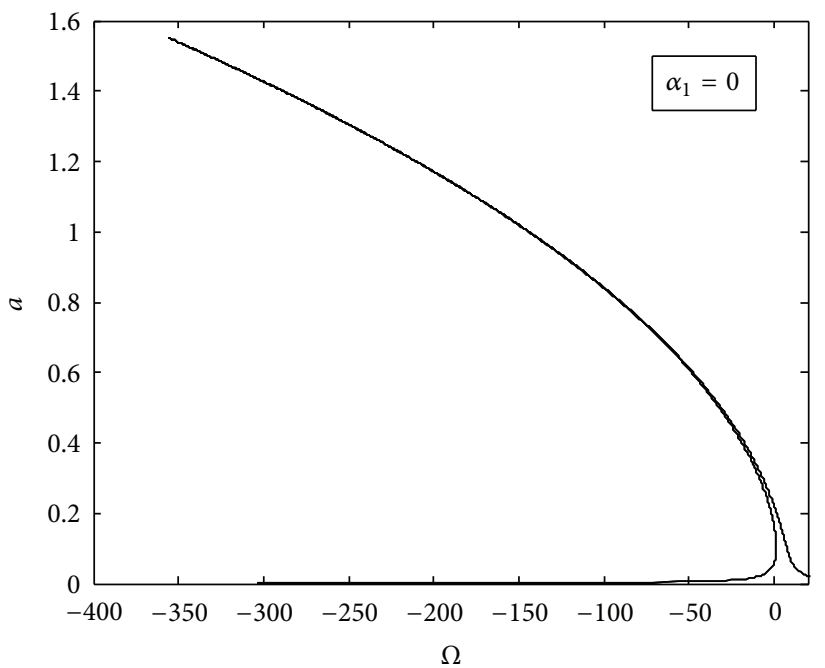

(a)

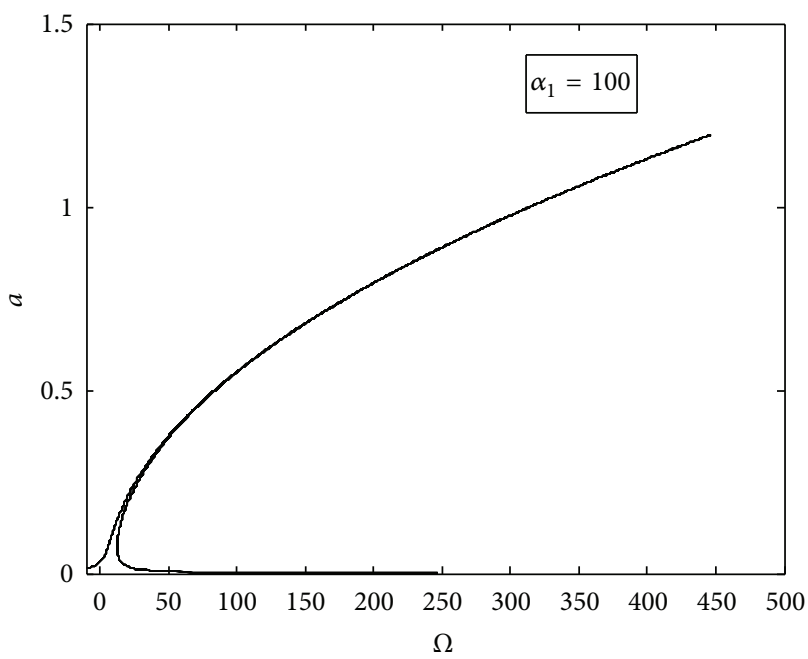

(c)

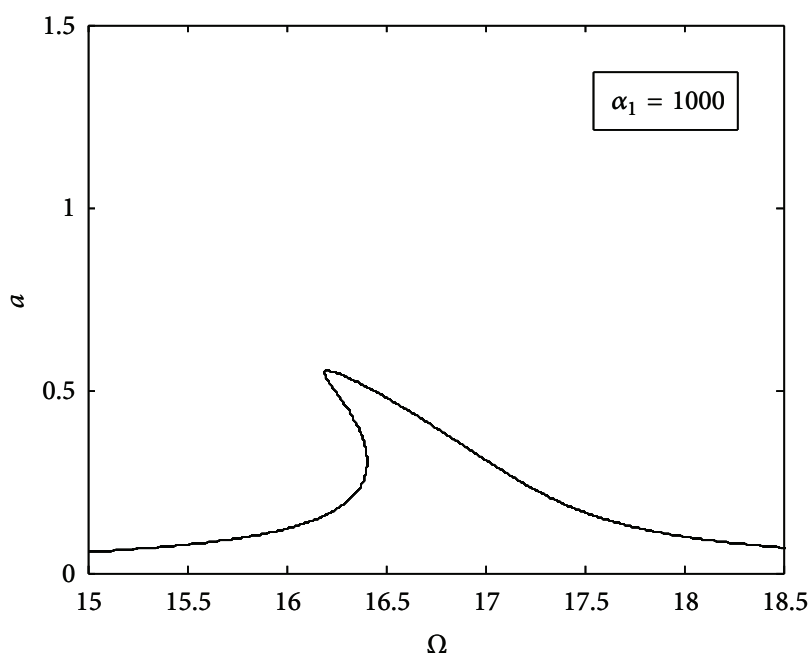

(e)

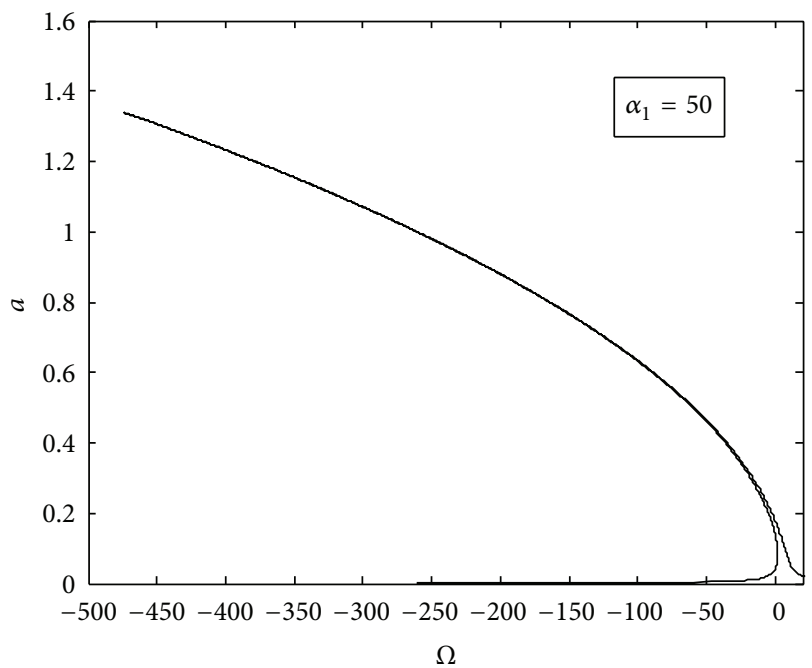

(b)

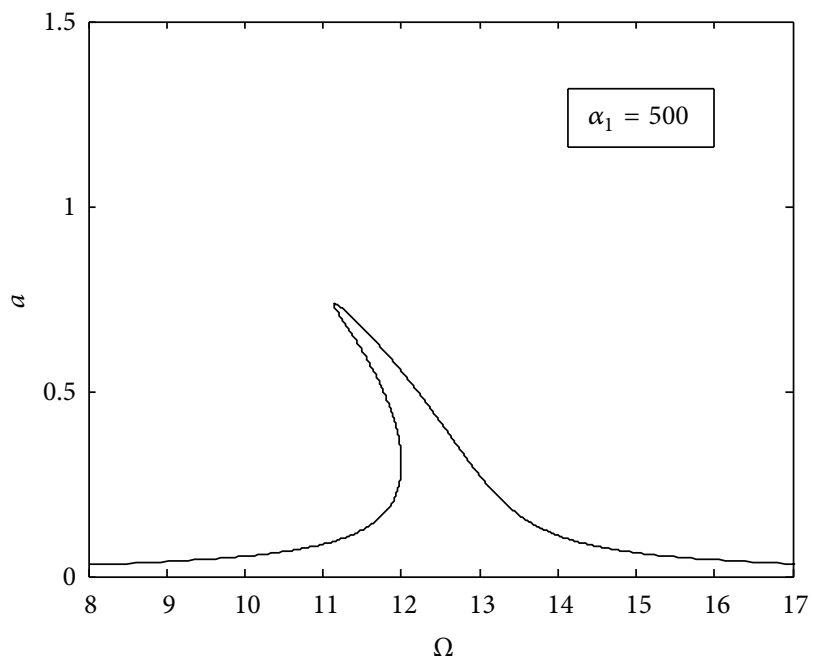

(d)

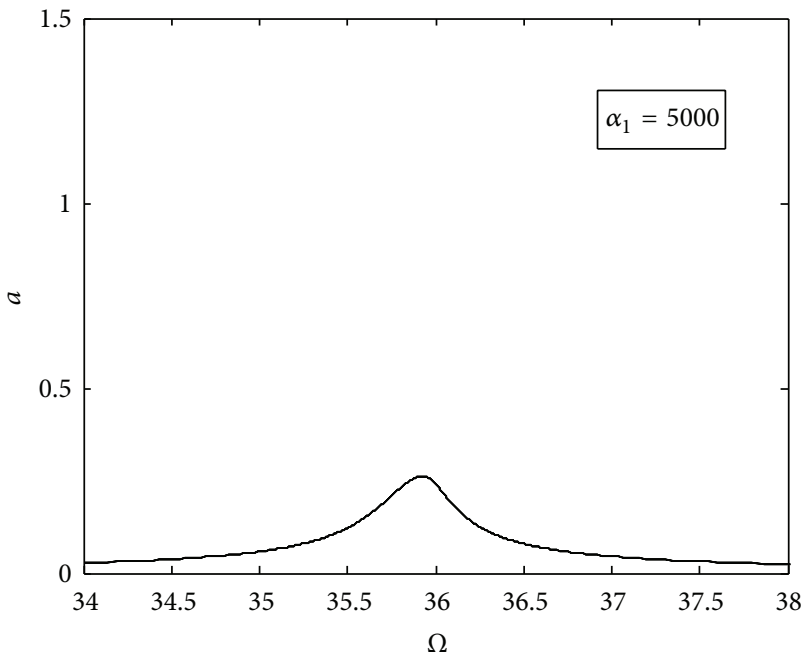

(f)

FIGURE 9: Frequency-response curves for the first mode: $\varepsilon=1, \mu=0.4, \alpha_{2}=10, \alpha_{3}=1, V_{\mathrm{AC}}=10, N=0, \Gamma=15$, and $B_{1}=0$. (a) $\alpha_{1}=0$, (b) $\alpha_{1}=50$, (c) $\alpha_{1}=100$, (d) $\alpha_{1}=500$, (e) $\alpha_{1}=1000$, and (f) $\alpha_{1}=5000$. 


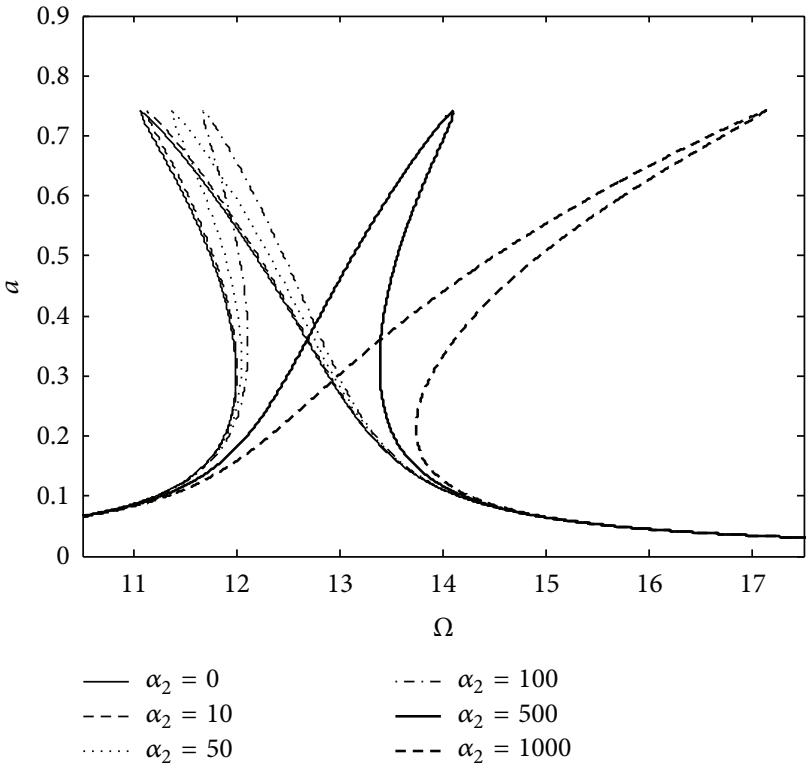

FIGURE 10: Frequency-response curves for the first mode: $\varepsilon=1, \mu=$ $0.4, \alpha_{1}=500, \alpha_{3}=1, V_{\mathrm{AC}}=10, N=0, \Gamma=15$, and $B_{1}=0$.

Equation (32) represents the modulations in the complex amplitudes which can be written in the polar form:

$$
A=\frac{1}{2} a e^{i \beta} .
$$

Substituting this form into (32) and separating real and imaginary parts, one finally obtains

$$
\begin{gathered}
\gamma^{\prime}=\sigma-\frac{M}{2 \omega_{n}}-\frac{a^{2} \lambda}{8 \omega_{n}}+\frac{\alpha_{3}}{4 a \omega_{n}} b_{6} V_{\mathrm{AC}}^{2} \cos \gamma, \\
a^{\prime}=-a \mu+\frac{\alpha_{3}}{4 \omega_{n}} b_{6} V_{\mathrm{AC}}^{2} \sin \gamma,
\end{gathered}
$$

where $\gamma$ is defined to be

$$
\gamma=\sigma T_{2}-\theta
$$

The response is finally found by substituting (37), (34), (29), (24), (23), and (14) into (9) and is

$$
\begin{aligned}
w(x, t)= & \varepsilon a \cos (2 \Omega t-\gamma) \sqrt{2} \sin n \pi x \\
& +\varepsilon^{2}\left(\frac{a^{2}}{2}\right)\left(\cos (4 \Omega t-2 \gamma) \phi_{1}(x)+\phi_{2}(x)\right) \\
& +O\left(\varepsilon^{3}\right) .
\end{aligned}
$$

The amplitude " $a$ " and the phase " $\gamma$ " are now governed by (35) and (36).

\section{Numerical Results}

In this section, we determine the nonlinear frequencies $\omega$, the effective nonlinearity parameter $\lambda$ of the system, and the frequency-response curves. Numerical results for free vibrations are presented first. Then forced vibrations with damping are considered.
4.1. Free Vibrations. Natural frequencies were given in (16),

$$
\omega=\sqrt{\beta^{2}+\alpha_{1}}
$$

and substituting for $\beta$ from (20) and (21) yields

$$
\begin{gathered}
\omega=\sqrt{\frac{3}{2} \pi^{4}+N \pi^{2}+\alpha_{1}} \quad n=1, B \neq 0, \\
\omega=\sqrt{n^{4} \pi^{4}+N n^{2} \pi^{2}+\alpha_{1}} \quad n=2,3,4 \ldots, B=0 .
\end{gathered}
$$

The first two frequency values with respect to the linear elastic foundations are given for various axial loads in Figure 2. As the axial load increases, the natural frequencies increase. Similarly, a gradual small increase of frequencies is observed as the linear elastic coefficient increases.

Next the undamped nonlinear frequency corrections to these linear ones, which are amplitude dependent, are calculated. Returning to (35) and (36), one takes $\sigma=0, \mu=$ $0, V_{\mathrm{AC}}=0$, and $\gamma=-\theta$. From (36) one obtains $a=a_{0}, a$ constant amplitude. Substituting this further into (35), using (37) yields

$$
\theta^{\prime}=\frac{a^{2} \lambda}{8 \omega_{n}}+\frac{M}{2 \omega_{n}} .
$$

The nonlinear frequency is

$$
\omega_{n l}=\omega_{n}+\varepsilon^{2}\left(\frac{M}{2 \omega_{n}}+\frac{a^{2} \lambda}{8 \omega_{n}}\right) .
$$

The first correction term is due to the nonideal boundary condition and the second term is due to the cumulative effect of all nonlinearities.

In Tables 2 and $3, \lambda$ values are given for cases $n=1$ and $n=2$, respectively, for various linear and nonlinear elastic foundation coefficients. From Table 2 , for the first mode $(n=$ 1 ), for a fixed lower value of the nonlinear elastic coefficient $\alpha_{2}$, a switch from softening to hardening and then hardening to softening behavior and final to hardening behavior is observed as linear elastic foundation coefficient $\alpha_{1}$ gradually increases. For sufficiently high values of $\alpha_{2}$, however, there is only one switch from softening to hardening behavior. From Table 3, for the second mode $(n=2)$, the switch between hardening and softening behavior cannot be observed. For this mode, only hardening behavior is obtained.

In Tables 4 and 5, $\lambda$ values are given for cases $n=1$ and $n=2$, respectively, for various linear elastic foundation coefficients and axial loadings. From these tables, for the first mode $(n=1)$, for some values of $\alpha_{1}$ and $N$ softening behavior can be observed (negative $\lambda$ ), whereas for the second mode $(n=2)$ only hardening behavior is observed.

From Table 4 , for the first mode $(n=1)$, for sufficiently high values of the axial loads (i.e., $N=500$ ), only hardening behavior is observed. For lower axial loading, switches from softening to hardening and vice versa can be observed depending on the numerical values of the elastic foundation coefficients $\alpha_{1}$ and axial loads $N$. From Table 5, for the second mode $(n=2)$ there is only hardening behavior. 


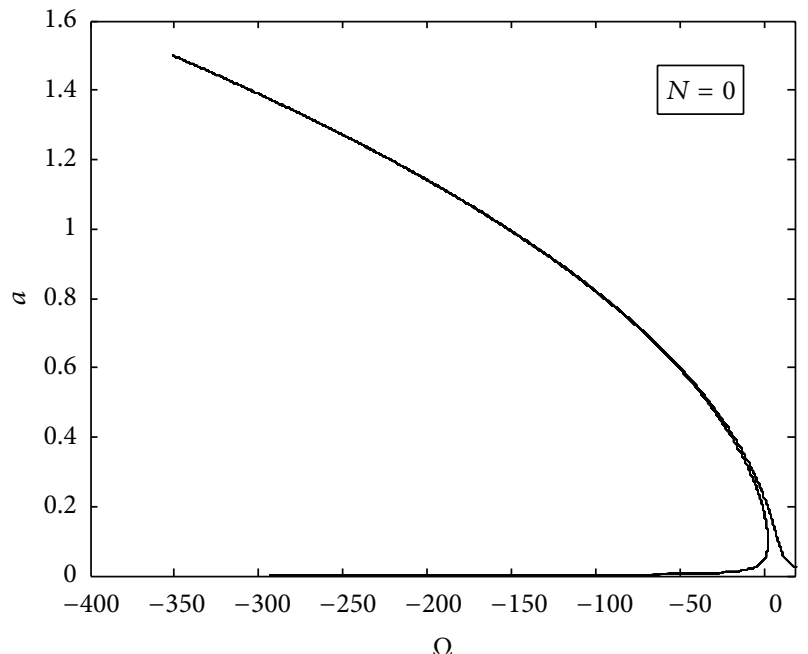

(a)

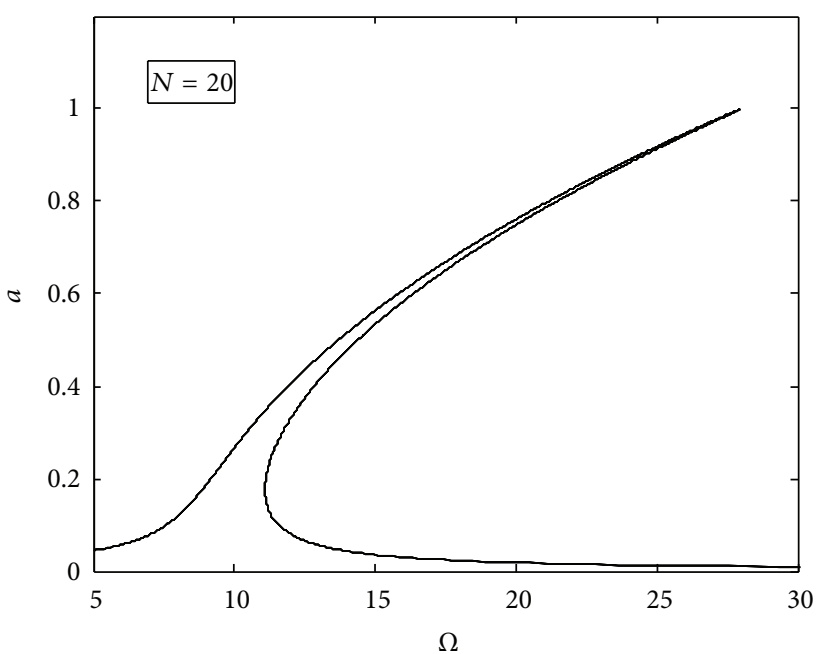

(c)

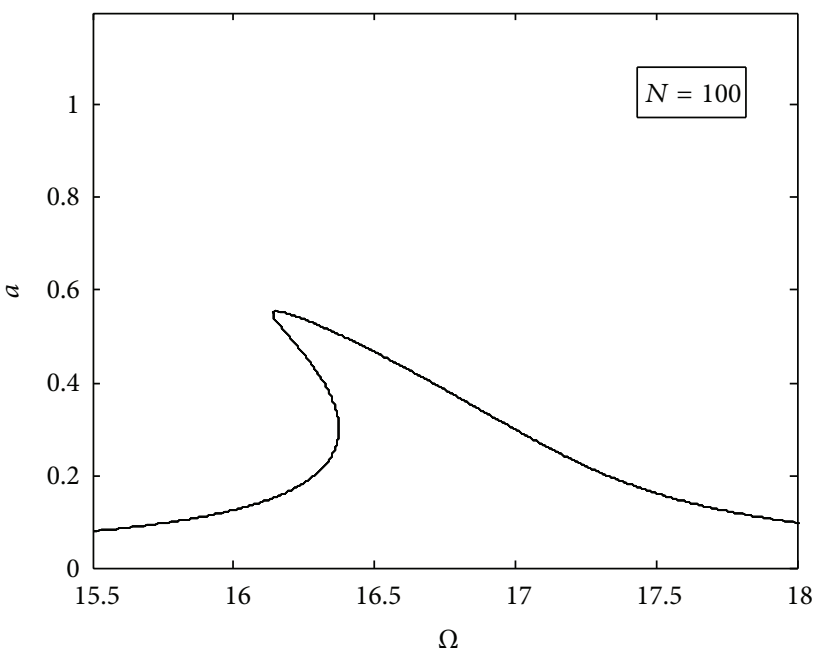

(e)

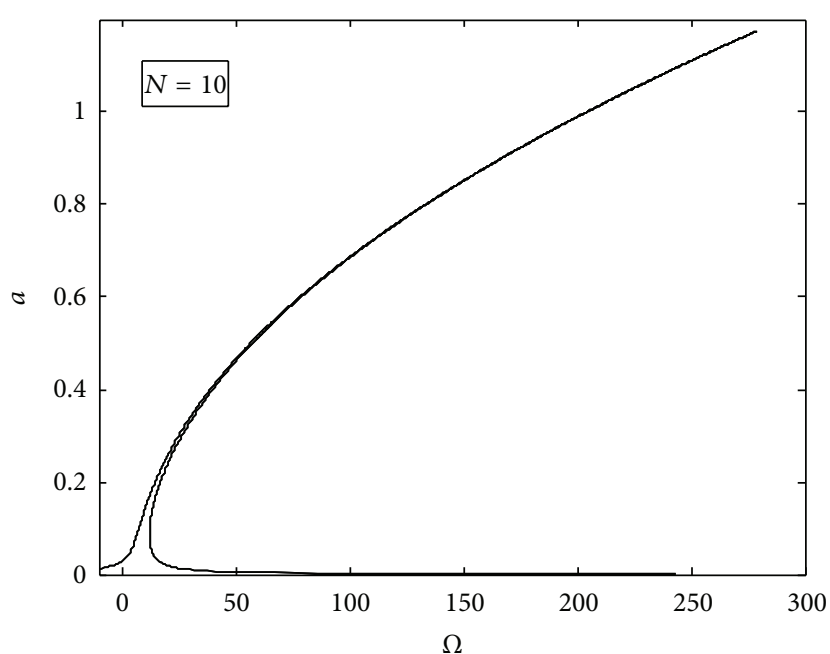

(b)

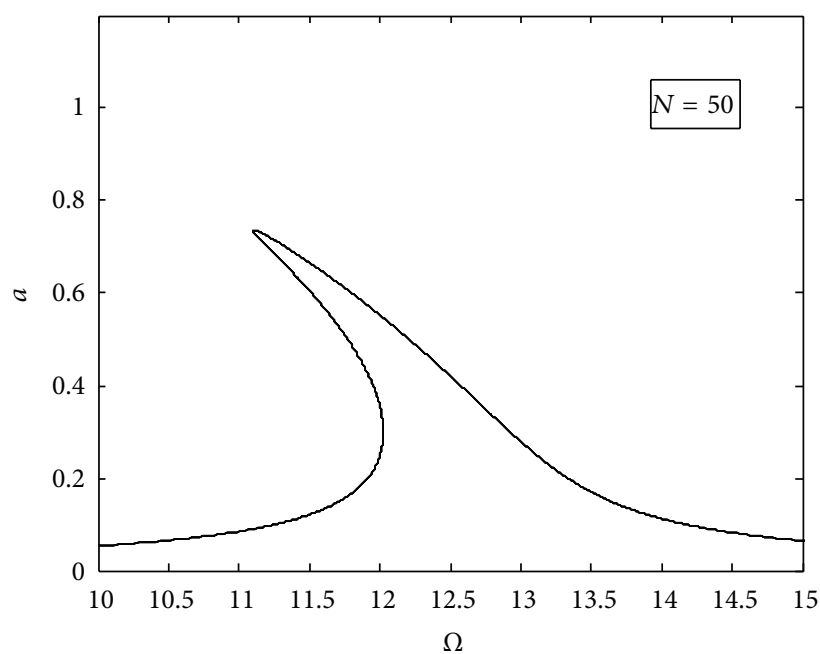

(d)

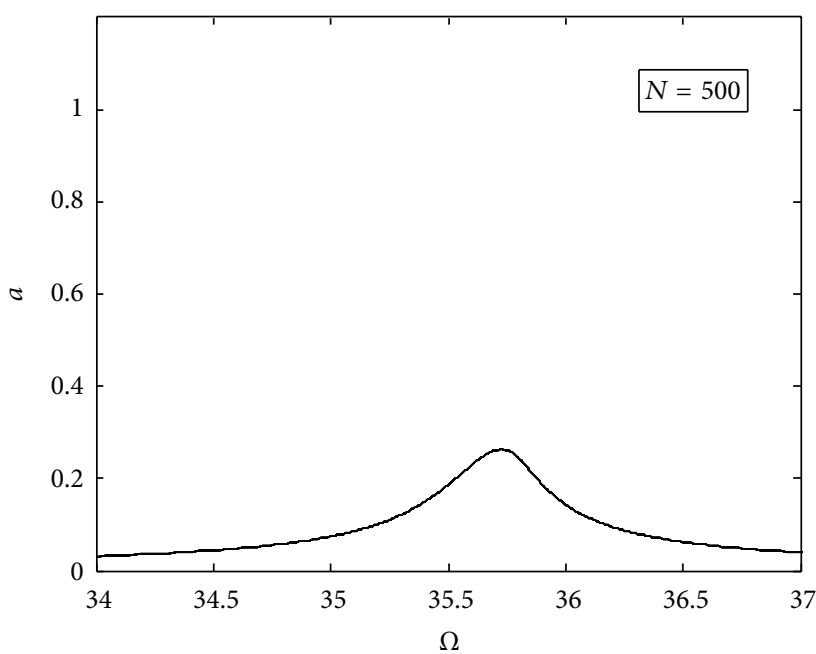

(f)

FIGURE 11: Frequency-response curves for the first mode: $\varepsilon=1, \mu=0.4, \alpha_{1}=10, \alpha_{2}=0, \alpha_{3}=1, V_{\mathrm{AC}}=10, \Gamma=15$, and $B_{1}=0$. (a) $N=0$, (b) $N=10$, (c) $N=20$, (d) $N=50$, (e) $N=100$, and (f) $N=500$. 
TABLE 2: $\lambda$ values corresponding to $\alpha_{1}$ and $\alpha_{2}$ values for $n=1, N=0$, and $\Gamma=15$.

\begin{tabular}{|c|c|c|c|c|c|c|}
\hline \multirow{2}{*}{$\alpha_{2}$} & \multicolumn{6}{|c|}{$\alpha_{1}$} \\
\hline & 0 & 50 & 100 & 500 & 1000 & 5000 \\
\hline 0 & -29136.78 & -60113.23 & 76892.06 & -1219.66 & -1321.26 & 868.78 \\
\hline 10 & -29091.78 & -60068.23 & 76937.06 & -1174.66 & -1276.25 & 913.78 \\
\hline 50 & -28911.78 & -59888.23 & 77117.06 & -994.66 & -1096.26 & 1093.78 \\
\hline 100 & -28686.78 & -59663.23 & 77342.06 & -769.66 & -871.26 & 1318.78 \\
\hline 500 & -26886.78 & -57863.23 & 79142.06 & 1030.335 & 928.74 & 3118.78 \\
\hline 1000 & -24636.78 & -55613.23 & 81392.06 & 3280.34 & 3178.74 & 5368.78 \\
\hline
\end{tabular}

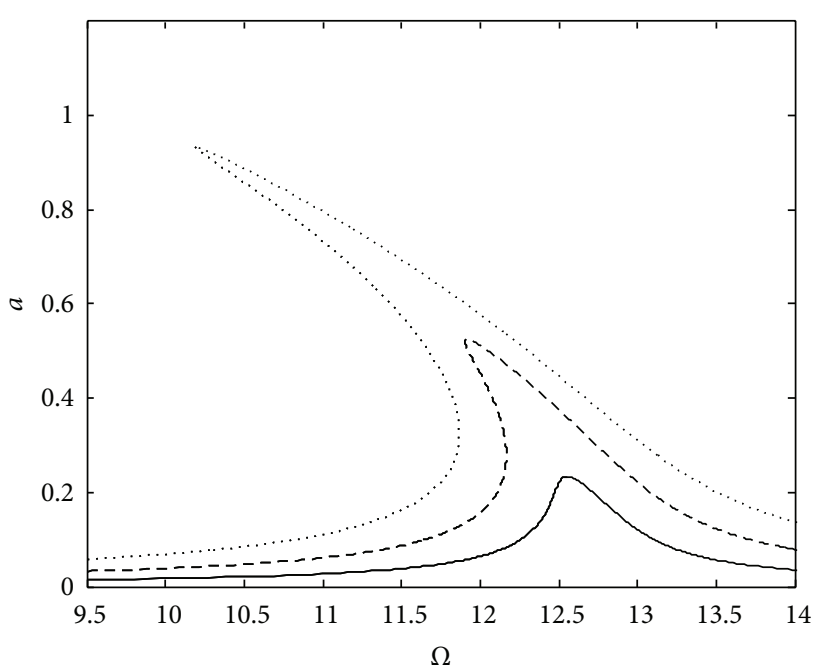

$$
\begin{aligned}
- & V_{\mathrm{AC}}=10 \\
---V_{\mathrm{AC}} & =15 \\
\ldots . . V_{\mathrm{AC}} & =20
\end{aligned}
$$

FIGURE 12: Frequency-response curves for the first mode: $\varepsilon=1, \mu=$ $0.4, \alpha_{1}=500, \alpha_{2}=10, \alpha_{3}=0.1, N=0, \Gamma=15$, and $B_{1}=0$.

TABLE 3: $\lambda$ values corresponding to $\alpha_{1}$ and $\alpha_{2}$ values for $n=2, N=0$, and $\Gamma=15$.

\begin{tabular}{lcccccc}
\hline \multirow{2}{\alpha}{$\alpha_{2}$} & \multicolumn{6}{c}{$\alpha_{1}$} \\
& 0 & 50 & 100 & 500 & 1000 & 5000 \\
\hline 0 & 16019.07 & 17151.16 & 18158.90 & 23442.61 & 26739.84 & 32555.18 \\
10 & 16064.07 & 17196.16 & 18203.90 & 23487.61 & 26784.84 & 32600.18 \\
50 & 16244.07 & 17376.16 & 18383.90 & 23667.61 & 26964.84 & 32780.18 \\
100 & 16469.07 & 17601.16 & 18608.90 & 23892.61 & 27189.84 & 33005.18 \\
500 & 18269.07 & 19401.16 & 20408.90 & 25692.61 & 28989.84 & 34805.18 \\
1000 & 20519.07 & 21651.16 & 22658.90 & 27942.61 & 31239.84 & 37055.18 \\
\hline
\end{tabular}

In Figures 3-5 the nonlinear frequencies versus amplitudes are shown for the first mode $(n=1)$. Figure 3 shows the comparison of nonlinear frequencies for various linear elastic foundation values. From this figure, since $\alpha_{2}=10$, $N=0, \Gamma=15$ from Table 2, the transitions from softening to hardening are expected between $\alpha_{1}=50$ and $\alpha_{1}=100$, $\alpha_{1}=1000$ and $\alpha_{1}=5000$. Mathematically speaking, since the dependence of $\lambda$ on $\alpha_{1}$ is rather complex and $\lambda$ changes sign depending on the values of $\alpha_{1}$, a frequent change of sign occurs which causes the transitions. However, as $\alpha_{2}$ becomes dominant on which $\lambda$ is linearly dependent, the sign changes are restricted to only one transition from softening to hardening. Physically speaking, the hardening behavior due to nonlinear elastic coefficient suppresses all other softening behaviors, and becomes dominant for a reasonably large linear elastic coefficient. In Figure 4, linear elastic foundation, axial load, and mid plane stretching values are fixed and only one transition occurs. In Figure 4(a), from Table 2 for $\alpha_{1}=$ $500, \Gamma=15$, and $N=0$, the critical transition value for $\alpha_{2}$ is 271.04. As can be verified from the figure, for $\alpha_{2}<271.04$ the behavior is softening type and for $\alpha_{2}>271.04$ it is of hardening type. In Figure 4(b), from Table 2 for $\alpha_{1}=1000$, $\Gamma=15$ and $N=0$ the critical transition value for $\alpha_{2}$ is 293.61. As can be verified from the figure, for $\alpha_{2}<293.61$, the behavior is softening type, and for $\alpha_{2}>293.61$ it is of hardening type.

In Figure 5, linear and nonlinear elastic foundation and mid plane stretching values are fixed and axial force values are increased. From this figure, since $\alpha_{1}=10, \alpha_{2}=0$, and $\Gamma=$ 15 , from Table 4, the transitions from softening to hardening are expected between $N=0$ and $N=10, N=100$ and $N=500$. Mathematically speaking, since the dependence of $\lambda$ on $N$ is rather complex and $\lambda$ changes sign depending on the values of $N$, a frequent change of sign occurs which causes the transitions. Physically speaking, the hardening behavior due to a reasonable large axial force value suppresses all other softening behaviors and becomes dominant for a reasonably large linear elastic coefficient.

In Figures 6, 7, and 8, the nonlinear frequency versus amplitudes are shown for the second mode $(n=2)$. For nonnegative elastic foundation coefficient values one can observe only a hardening type of behavior.

4.2. Forced Vibrations with Damping. To consider forced vibrations with damping one returns again to the amplitude and phase modulation equations given in (35) and (36). For steady-state solutions $a^{\prime}=\gamma^{\prime}=0$. The frequency response equation is determined by eliminating $\gamma$ between (35) and (36), finding $\sigma$, and then substituting this value into (29)

$$
2 \Omega=\omega_{n}+\varepsilon^{2}\left(\frac{1}{2 \omega_{n}} M+\frac{1}{8 \omega_{n}} a^{2} \lambda \mp \sqrt{\frac{\alpha_{3}^{2} b_{6}^{2} V_{\mathrm{AC}}^{4}}{16 \omega_{n}^{2} a^{2}}-\mu^{2}}\right) .
$$




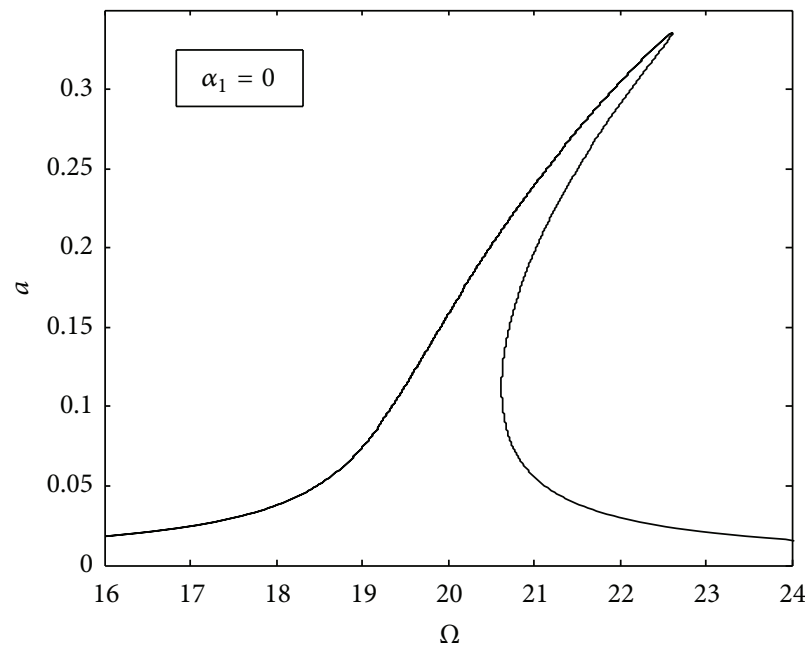

(a)

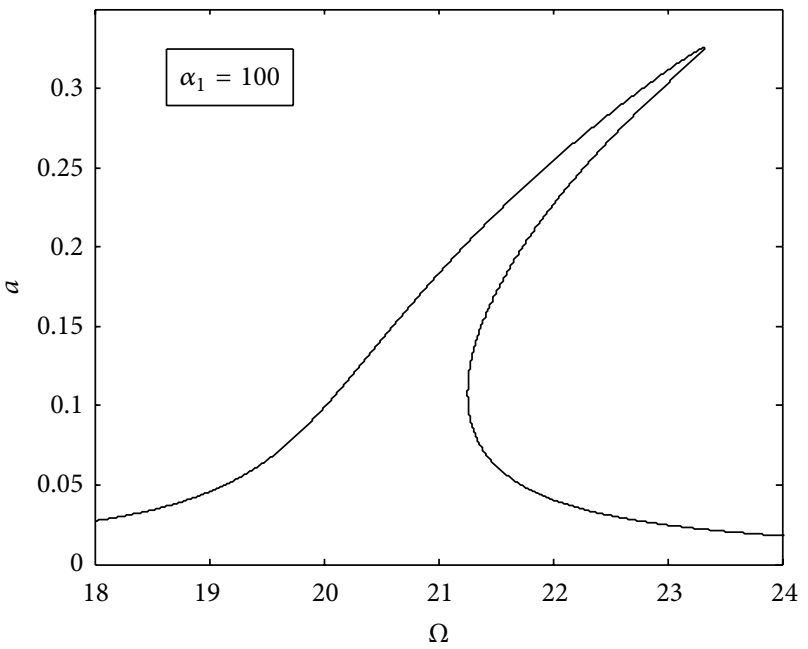

(c)

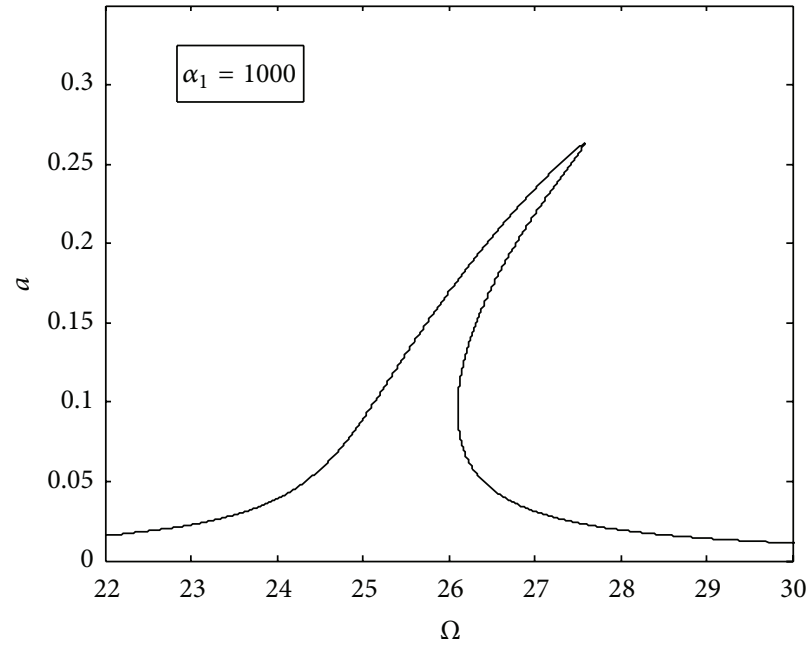

(e)

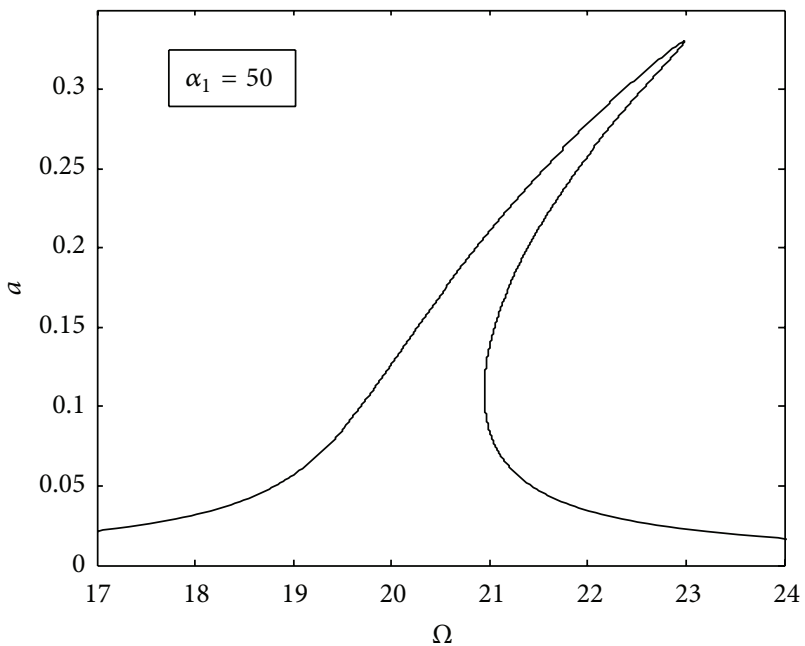

(b)

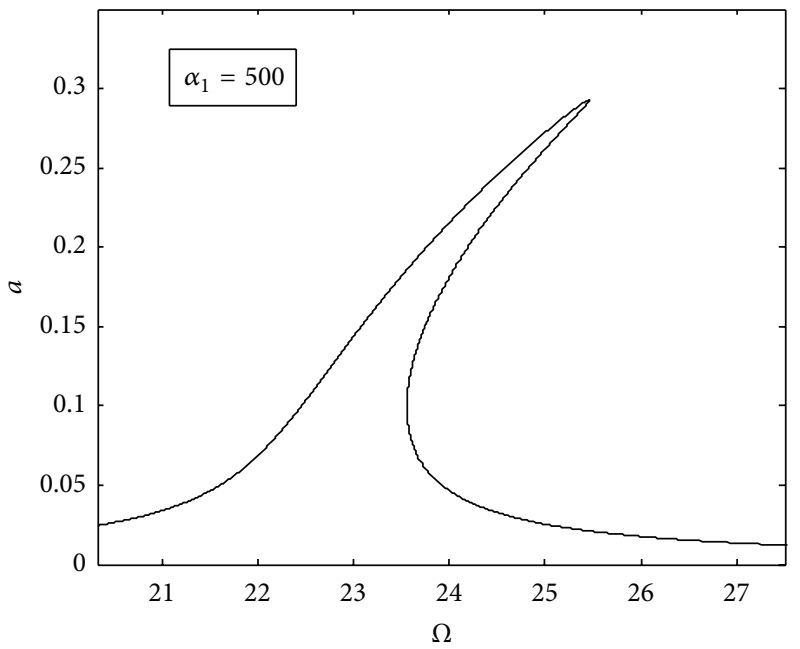

(d)

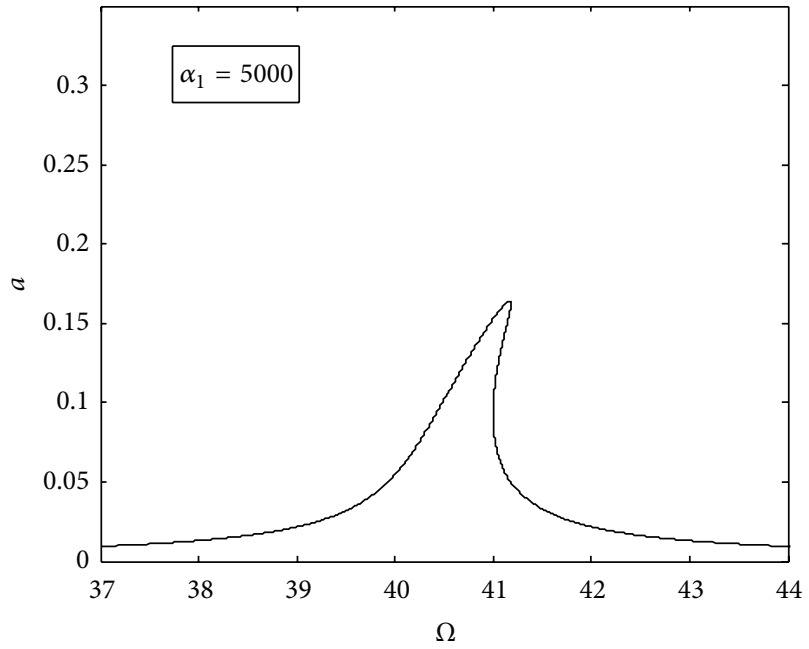

(f)

FIGURE 13: Frequency-response curves for the second mode: $\varepsilon=1, \mu=0.4, \alpha_{2}=10, \alpha_{3}=0.5, V_{\mathrm{AC}}=10, N=0, \Gamma=15$, and $B_{1}=0$. (a) $\alpha_{1}=0$, (b) $\alpha_{1}=50$, (c) $\alpha_{1}=100$, (d) $\alpha_{1}=500$, (e) $\alpha_{1}=1000$, and (f) $\alpha_{1}=5000$. 
TABLE 4: $\lambda$ values corresponding to $\alpha_{1}$ and $N$ values for $n=1, \alpha_{2}=0$, and $\Gamma=15$.

\begin{tabular}{|c|c|c|c|c|c|c|c|}
\hline \multirow{2}{*}{$N$} & \multicolumn{7}{|c|}{$\alpha_{1}$} \\
\hline & 0 & 10 & 50 & 100 & 500 & 1000 & 5000 \\
\hline 0 & -29136.78 & -31769.73 & -60113.23 & 76892.06 & -1219.66 & -1321.26 & 868.78 \\
\hline 10 & 83207.07 & 50114.0 & 16068.78 & 6458.78 & -1448.28 & -1221.06 & 889.82 \\
\hline 20 & 6599.75 & 5598.68 & 2890.98 & 1060.28 & -1508.12 & -1117.22 & 910.22 \\
\hline 50 & -1194.90 & -1232.26 & -1349.87 & -1441.49 & -1327.62 & -812.96 & 967.78 \\
\hline 100 & -1333.93 & -1324.23 & -1284.49 & -1233.26 & -819.36 & -381.04 & 1053.11 \\
\hline 500 & 854.5 & 856.71 & 865.47 & 876.27 & 956.78 & 1044.65 & 1461.58 \\
\hline
\end{tabular}

TABLE 5: $\lambda$ values corresponding to $\alpha_{1}$ and $N$ values for $n=2, \alpha_{2}=0$, and $\Gamma=15$.

\begin{tabular}{lccccccc}
\hline$N$ & \multicolumn{2}{c}{$\alpha_{1}$} & 500 & 1000 & 5000 \\
\hline 0 & 0 & 10 & 16256.52 & 17151.16 & 18158.90 & 23442.61 & 26739.84 \\
10 & 16019.07 & 18067.87 & 18793.60 & 19619.91 & 24114.06 & 27066.34 & 32555.18 \\
20 & 17886.48 & 19588.35 & 20187.61 & 20875.91 & 24735.88 & 27385.82 & 32598.16 \\
50 & 19431.12 & 22904.88 & 23272.85 & 23703.36 & 26287.35 & 28250.30 & 32672.56 \\
100 & 22809.36 & 26130.31 & 26328.56 & 26564.61 & 28082.72 & 29367.79 & 32803.64 \\
500 & 26079.37 & 32216.14 & 32236.34 & 32261.20 & 32445.67 & 32645.38 & 33570.82 \\
\hline
\end{tabular}

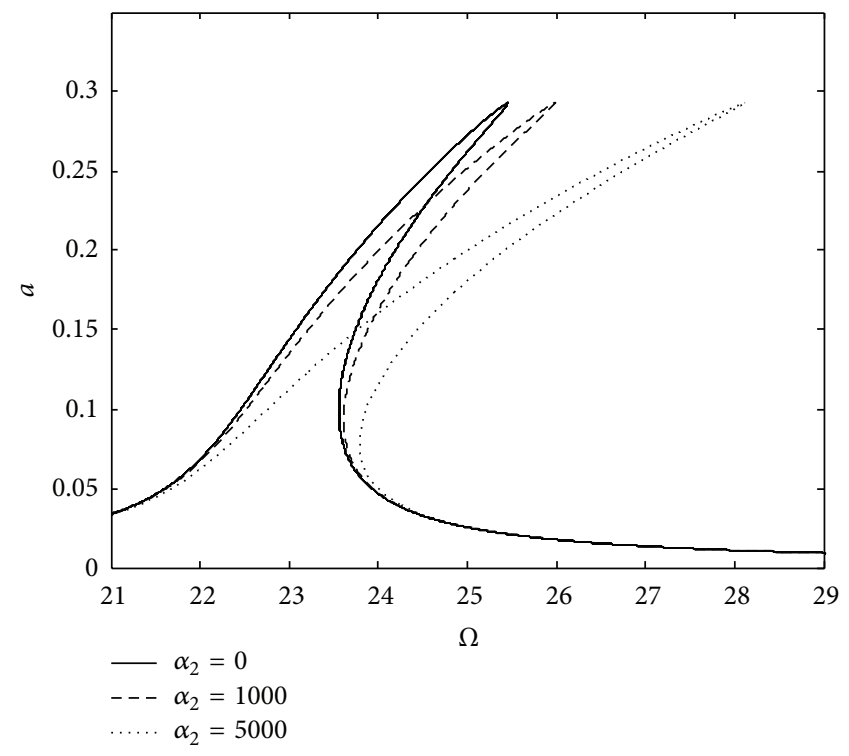

FIGURE 14: Frequency-response curves for the second mode: $\varepsilon=$ $1, \mu=0.4, \alpha_{1}=500, \alpha_{3}=0.5, V_{\mathrm{AC}}=10, N=0, \Gamma=15$, and $B_{1}=$ 0 .

In this section, to describe the dynamic response of the microbeam, frequency-response curves are analyzed with respect to the effective physical parameters which are the linear and nonlinear elastic foundation coefficients, the voltage amplitudes, the axial forces, and the strength of the midplane stretching. In Figures 9-11, for the first mode, the frequency-response curves are shown with changes of qualitative behavior from softening to hardening.

In Figure 9, frequency-response curves are drawn for the specific values of linear elastic foundation parameter $\alpha_{1}$ for $\alpha_{2}=10, N=0$, and $\Gamma=15$. As the linear elastic foundation increases, the frequency-response curves shift to the right with the changes of qualitative behavior from softening to hardening. These changes are observed between $\alpha_{1}=50$ and $\alpha_{1}=100, \alpha_{1}=1000$ and $\alpha_{1}=5000$.

Effect of nonlinear elastic foundation coefficient on the frequency-response curves is depicted in Figure 10. For increasing nonlinear elastic foundation coefficients, transition occurs from softening behavior to hardening behavior without an increase in the maximum amplitudes. In Figure 11, the frequency-response curves are drawn for specific values of the axial force parameter $N$. As the axial force increases, the frequency curves shift to the right with a decrease in the peak amplitudes. The transitions from softening to hardening are observed between $N=0$ and $N=10, N=100$ and $N=500$.

It is observed that the effects of linear elastic foundation coefficients on frequency-response curves are similar to the effects of the axial load coefficients, when Figures 9 and 11 are addressed together. Their variations affect maximum amplitudes of the frequency-response curves and shift these curves. In the frequency-response curves, two transitions from softening to hardening are obtained.

In Figure 12, frequency-response curves are drawn for specific values of the voltage amplitude $V_{\mathrm{AC}}$ for $\alpha_{1}=500$, $\alpha_{2}=10, N=0$, and $\Gamma=15$. Note that $\alpha_{3}$ and $V_{\mathrm{AC}}$ appear as multiplications in the equations of motion so due to their similar influences only $V_{\mathrm{AC}}$ effect is considered. The maximum amplitudes increase for an increase in $V_{\mathrm{AC}}$ and the multivalued regions responsible for jump phenomena increase considerably.

In Figures 13-15, the frequency-response curves are shown for the second mode. In Figure 13, $\alpha_{2}$ and $N$ are fixed and $\alpha_{1}$ is increased. All curves show hardening behavior. In Figure 14, $\alpha_{1}$ and $N$ are fixed and $\alpha_{2}$ is increased. The multivalued regions increase without an increase in the maximum amplitude. In Figure 15, the frequency-response curves are 


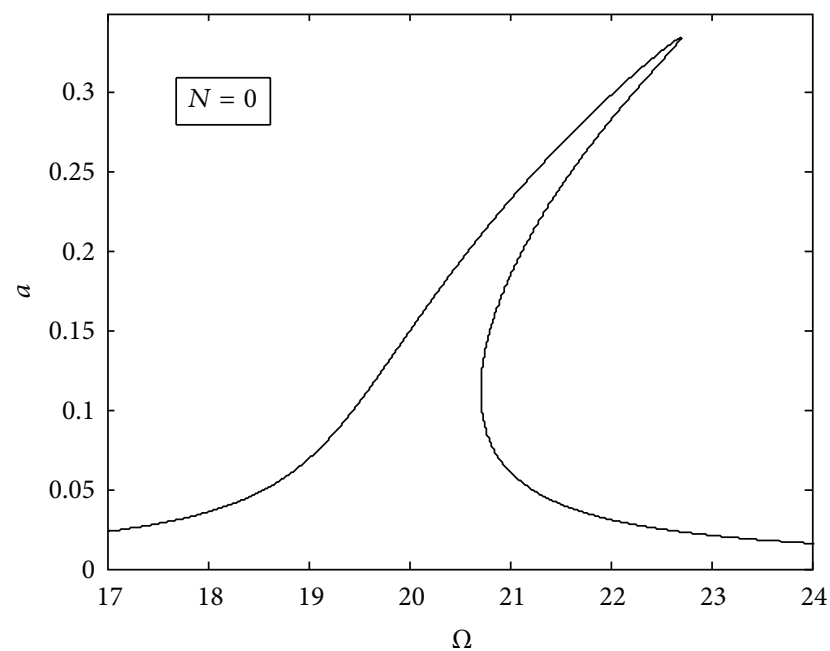

(a)

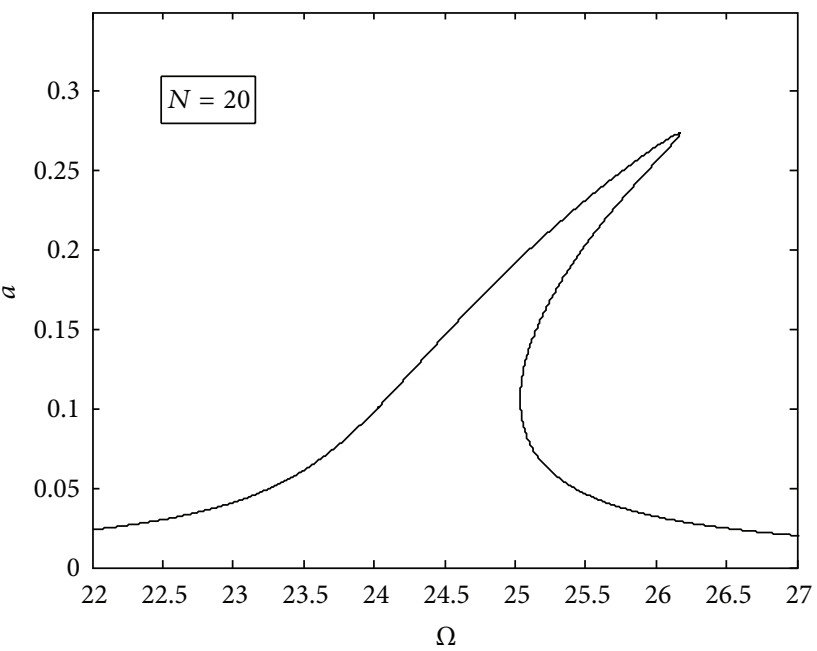

(c)

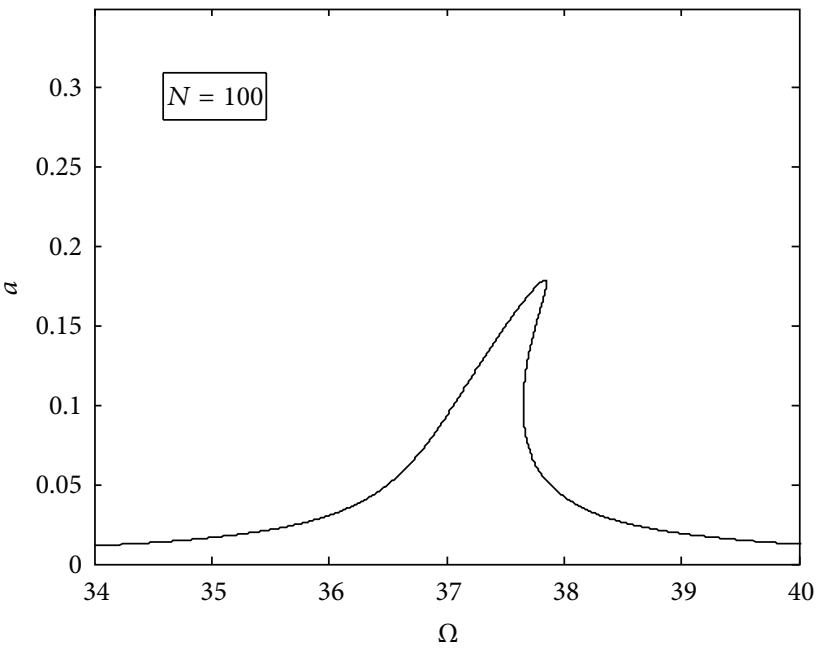

(e)

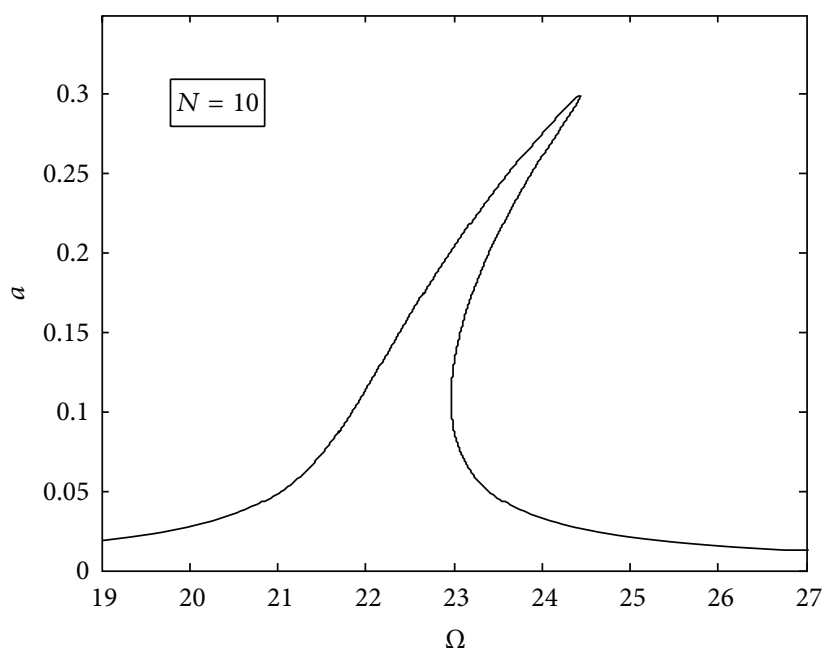

(b)

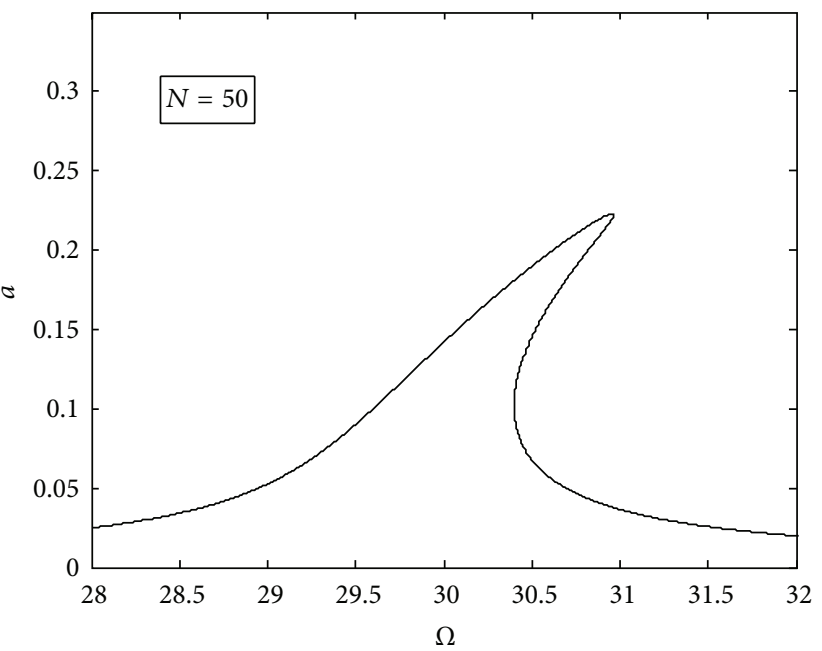

(d)

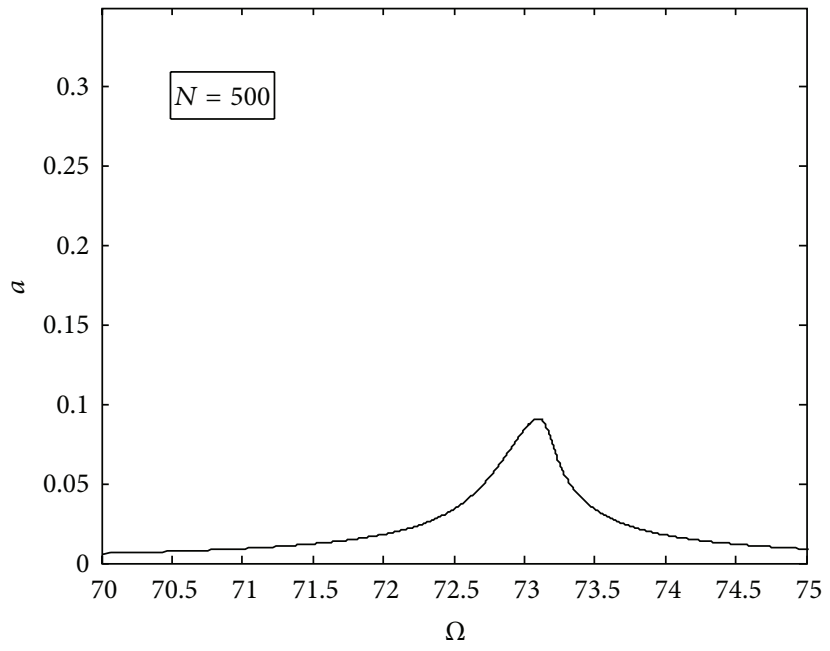

(f)

FIGURE 15: Frequency-response curves for the second mode: $\varepsilon=1, \mu=0.4, \alpha_{1}=10, \alpha_{2}=0, \alpha_{3}=0.5, V_{\mathrm{AC}}=10, \Gamma=15$, and $B_{1}=0$. (a) $N=0$, (b) $N=10$, (c) $N=20$, (d) $N=50$, (e) $N=100$, and (f) $N=500$. 


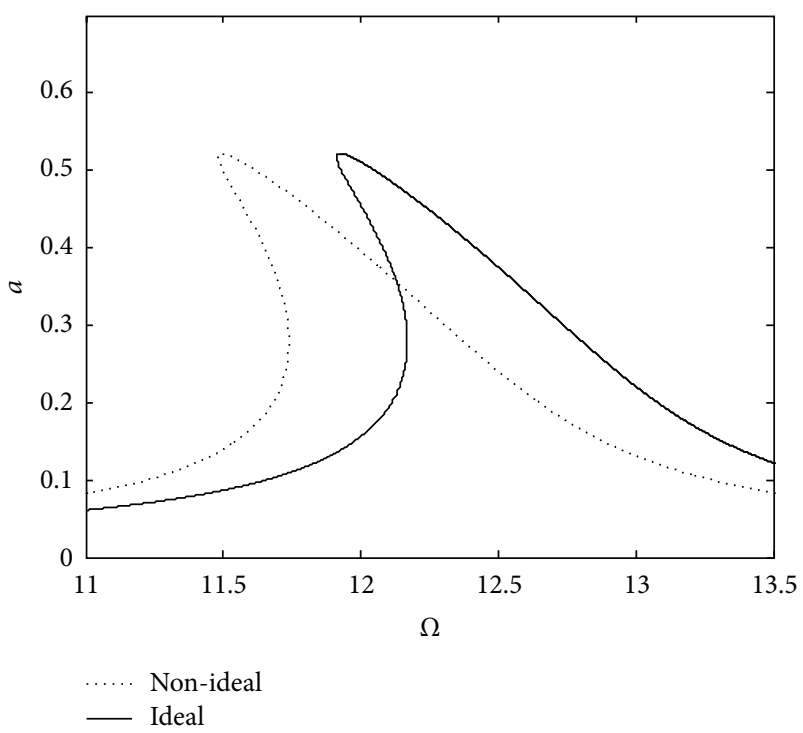

(a)

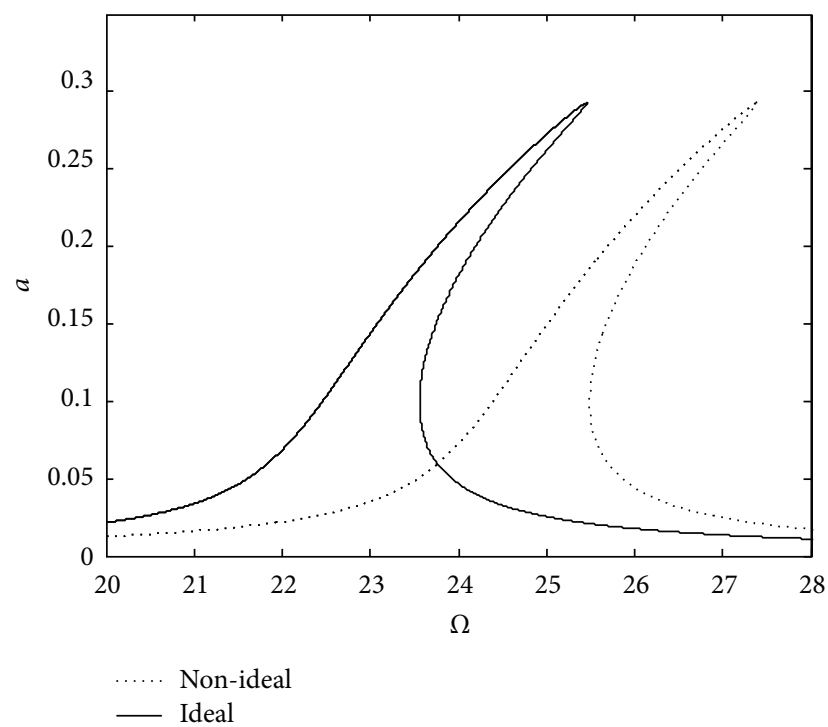

(b)

FIGURE 16: Frequency-response curves for ideal (solid) and nonideal (dotted) cases: (a) first mode $\left(\varepsilon=1, \alpha_{1}=500, \alpha_{2}=10, \alpha_{3}=0.5, N=0\right.$, $V_{\mathrm{AC}}=10, \mu=0.4, \Gamma=15$, and $\left.B_{1}=1\right)$, (b) second mode $\left(\varepsilon=1, \alpha_{1}=500, \alpha_{2}=10, \alpha_{3}=0.5, N=0, V_{\mathrm{AC}}=10, \mu=0.4, \Gamma=15\right.$, and $\left.B_{1}=1\right)$.

drawn for specific values of the axial force parameter $N$. As the axial force increases, the frequency curves shift to the right with a decrease in the maximum amplitudes.

The designers may use the frequency-response curves given in Figures 9-15 to select the optimum values of linearnonlinear elastic foundation coefficients, voltage amplitudes, and axial loads for their design.

4.3. Effect of the Nonideal Boundary Condition. Response amplitude versus excitation frequency curves is drawn for ideal (solid) and nonideal (dashed) cases for the first mode in Figure 16(a) and for the second mode in Figure 16(b). It can be seen from these graphs that the nonideal frequencyresponse curves shift to the left with respect to the ideal case for the first mode. On the contrary, for the second mode, the shift is to the right. Resonance shifting is a very important phenomenon, especially in resonant microbeams. It can be achieved by transition from softening to hardening behavior as well as by shifting the curves with nonidealities.

It is significant to understand the nonlinear behavior of resonant microbeams in order to improve their design. Various design parameters, such as the curvature of the microbeam, the amplitude of the AC excitation voltages, the elastic foundation coefficients, and the nonideal boundary conditions, have to be investigated to optimize the resonant MEMS sensors and minimize any undesirable behavior.

Using the results of this work, frequency responses of the microbeams can be passively controlled by adjusting design parameters.

\section{Concluding Remarks}

The nonlinear dynamic response of a slightly curved microbeam resting on a nonlinear elastic foundation that is actuated by an electric load subject to an axial load is analyzed. The nonlinearities arise due to the nonlinear elastic foundation and immovable end conditions which cause midplane stretching. Nonideal boundary conditions are defined and formulated using perturbation theory. Approximate analytical solutions are found using the method of multiple scales, a perturbation technique.

Effects of the linear elastic foundation, the nonlinear elastic foundation, the AC harmonic load of amplitude, the axial force, and the midplane stretching on the responses are investigated. Nonlinear frequencies and frequency-response curves are drawn for various design parameters. Results show that increasing the linear elastic foundation coefficient shifts curves to the right with either hardening or softening behavior. For an increase in the nonlinear elastic foundation coefficient, the multivalued regions responsible for the jump phenomena increase considerably without an increase in the maximum amplitudes. Maximum amplitudes increase for higher AC harmonic loads of the amplitude.

Softening and hardening behavior can be observed for the first mode depending on the physical parameters. It is found that the effect of curvature is of softening type. For sufficiently high values of the coefficients, the elastic foundation and axial load may suppress the softening behavior resulting in a hardening behavior of the nonlinearity. For the second mode and for positive values of the elastic foundation coefficients, only hardening behavior can be observed. The curvature effects are of hardening type only.

The nonideal boundary conditions can cause shifting to the left or right hand side in the frequency amplitude curves, depending on the mode numbers. This may cause a shift of the resonant region which has direct practical implications in design of such systems. Depending on the goal of the design, by adjusting nonideal conditions, system can be run or avoided from running in the resonant region. 
Results provide a reference for the choice of reasonable resonant conditions, design, and industrial applications of such slightly curved microbeams bonded to an elastic foundation.

\section{References}

[1] M. I. Younis and A. H. Nayfeh, "A study of the nonlinear response of a resonant microbeam to an electric actuation," Nonlinear Dynamics, vol. 31, no. 1, pp. 91-117, 2003.

[2] W. Zhang and G. Meng, "Nonlinear dynamical system of microcantilever under combined parametric and forcing excitations in MEMS," Sensors and Actuators A, vol. 119, no. 2, pp. 291-299, 2005.

[3] R. M. C. Mestrom, R. H. B. Fey, J. T. M. van Beek, K. L. Phan, and H. Nijmeijer, "Modelling the dynamics of a MEMS resonator: simulations and experiments," Sensors and Actuators A, vol. 142, no. 1, pp. 306-315, 2008.

[4] X. L. Jia, J. Yang, S. Kitipornchai, and C. W. Lim, "Resonance frequency response of geometrically nonlinear micro-switches under electrical actuation," Journal of Sound and Vibration, vol. 331, no. 14, pp. 3397-3411, 2012.

[5] S. Abu-Salih and D. Elata, "Electromechanical buckling of a prestressed layer bonded to an elastic foundation," in Proceedings of the NSTI Nanotechnology Conference and Trade Show (NSTI Nanotech '04), pp. 223-226, March 2004.

[6] B. Rivlin and D. Elata, "Design of nonlinear springs for attaining a linear response in gap-closing electrostatic actuators," International Journal of Solid and Structures, vol. 49, no. 26, pp. 38163822, 2012.

[7] S. Kong, S. Zhou, Z. Nie, and K. Wang, "The size-dependent natural frequency of Bernoulli-Euler micro-beams," International Journal of Engineering Science, vol. 46, no. 5, pp. 427-437, 2008.

[8] H. M. Ouakad and M. I. Younis, "The dynamic behavior of MEMS arch resonators actuated electrically," International Journal of Non-Linear Mechanics, vol. 45, no. 7, pp. 704-713, 2010.

[9] J. Casals-Terre and A. Shkel, "Snap-action bistable micromechanism actuated by nonlinear resonance," in Proceedings of the 4th IEEE Conference on Sensors, pp. 893-896, Irvine, Calif, USA, November 2005.

[10] J. Qiu, J. H. Lang, and A. H. Slocum, "A curved-beam bistable mechanism," Journal of Microelectromechanical Systems, vol. 13, no. 2, pp. 137-146, 2004.

[11] Y. Zhang, Y. Wang, Z. Li, Y. Huang, and D. Li, "Snap-through and pull-in instabilities of an arch-shaped beam under an electrostatic loading," Journal of Microelectromechanical Systems, vol. 16, no. 3, pp. 684-693, 2007.

[12] S. Krylov, B. R. Ilic, D. Schreiber, S. Seretensky, and H. Craighead, "The pull-in behavior of electrostatically actuated bistable microstructures," Journal of Micromechanics and Microengineering, vol. 18, no. 5, pp. 055026-055046, 2008.

[13] K. Das and R. C. Batra, "Pull-in and snap-through instabilities in transient deformations of microelectromechanical systems," Journal of Micromechanics and Microengineering, vol. 19, no. 3, pp. 035008-035027, 2009.

[14] M. I. Younis, E. M. Abdel-Rahman, and A. Nayfeh, "A reducedorder model for electrically actuated microbeam-based MEMS," Journal of Microelectromechanical Systems, vol. 12, no. 5, pp. 672-680, 2003.
[15] L. Ruzziconi, A. M. Bataineh, M. I. Younis, and S. Lenci, "Theoretical and experimental investigation of the nonlinear response of an electrically actuated imperfect microbeam," in Proceedings of the International Conference on Structural Nonlinear Dynamics and Diagnosis (CSNDD '12), vol. 1, 2012.

[16] L. Ruzziconi, M. I. Younis, and S. Lenci, "An electrically actuated imperfect microbeam: dynamical integrity for interpreting and predicting the device response," Meccanica, 2013.

[17] H. O. Ekici and H. Boyaci, "Effects of non-ideal boundary conditions on vibrations of microbeams," Journal of Vibration and Control, vol. 13, no. 9-10, pp. 1369-1378, 2007.

[18] H. R. Öz, M. Pakdemirli, E. Özkaya, and M. Yilmaz, "Nonlinear vibrations of a slightly curved beam resting on anonlinear elastic foundation," Journal of Sound and Vibration, vol. 212, no. 2, pp. 295-309, 1998.

[19] H. R. Öz and M. Pakdemirli, "Two-to-one internal resonances in a shallow curved beam resting on an elastic foundation," Acta Mechanica, vol. 185, no. 3-4, pp. 245-260, 2006.

[20] M. Pakdemirli and H. Boyaci, "Vibrations of a stretched beam with non-ideal boundary conditions," Mathematical and Computational Applications, vol. 6, no. 3, pp. 217-220, 2001.

[21] M. Pakdemirli and H. Boyaci, "Effect of non-ideal boundary conditions on the vibrations of continuous systems," Journal of Sound and Vibration, vol. 249, no. 4, pp. 815-823, 2002.

[22] M. Pakdemirli and H. Boyaci, "Non-linear vibrations of a simple-simple beam with a non-ideal support in between," Journal of Sound and Vibration, vol. 268, no. 2, pp. 331-341, 2003.

[23] M. Pakdemirli and H. Boyaci, "Vibrations of a simply supported beam with a non-ideal support at an intermediate point," Mathematical and Computational Applications, vol. 8, no. 1-3, pp. 159-164, 2003.

[24] D. J. Griffiths, Introduction to Electrodynamics, Prentice Hall, Englewood Cliffs, NJ, USA, 1981.

[25] A. H. Nayfeh, J. F. Nayfeh, and D. T. Mook, "On methods for continuous systems with quadratic and cubic nonlinearities," Nonlinear Dynamics, vol. 3, no. 2, pp. 145-162, 1992.

[26] M. Pakdemirli, S. A. Nayfeh, and A. H. Nayfeh, "Analysis of oneto-one autoparametric resonances in cables-Discretization vs. direct treatment," Nonlinear Dynamics, vol. 8, no. 1, pp. 65-83, 1995.

[27] M. Pakdemirli, "A comparison of two perturbation methods for vibrations of systems with quadratic and cubic nonlinearities," Mechanics Research Communications, vol. 21, no. 2, pp. 203-208, 1994.

[28] M. Pakdemirli and H. Boyaci, “The direct-perturbation method versus the discretization-perturbation method: linear systems," Journal of Sound and Vibration, vol. 199, no. 5, pp. 825-832, 1997.

[29] A. H. Nayfeh, Introduction to Perturbation Techniques, Wiley, New York, NY, USA, 1981. 


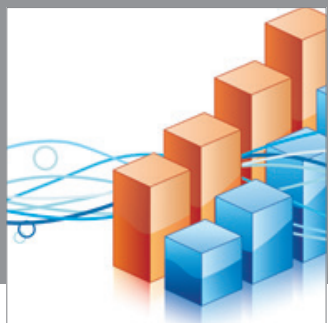

Advances in

Operations Research

mansans

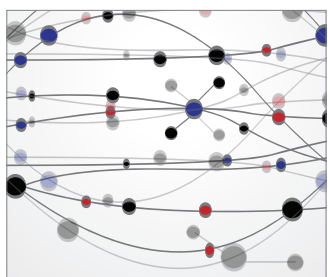

The Scientific World Journal
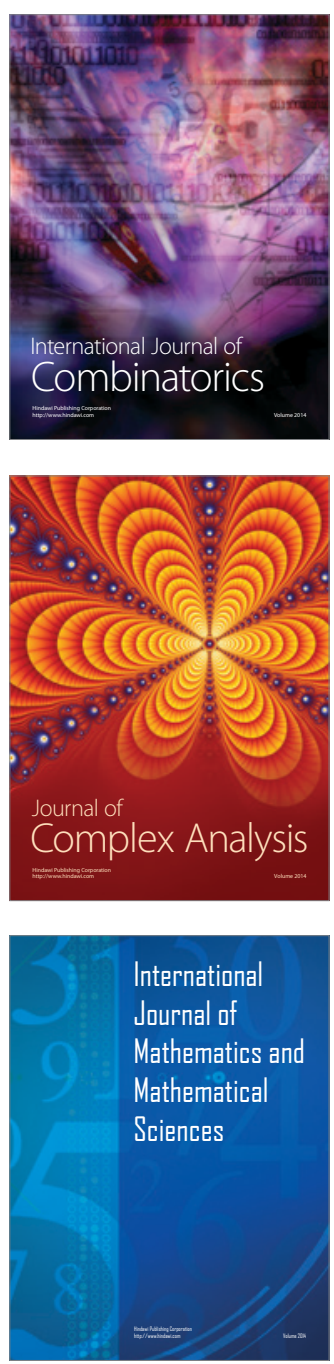
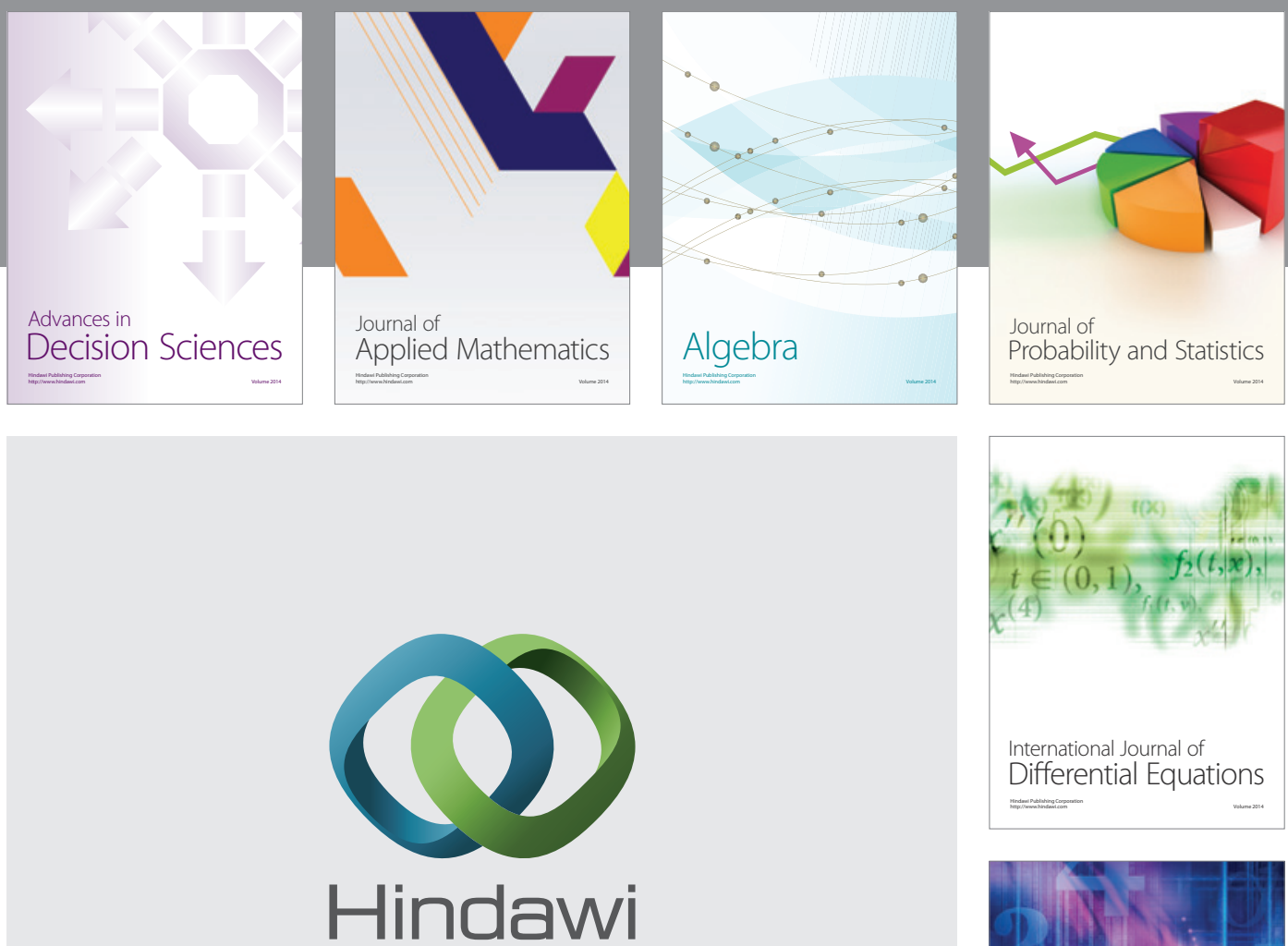

Submit your manuscripts at http://www.hindawi.com
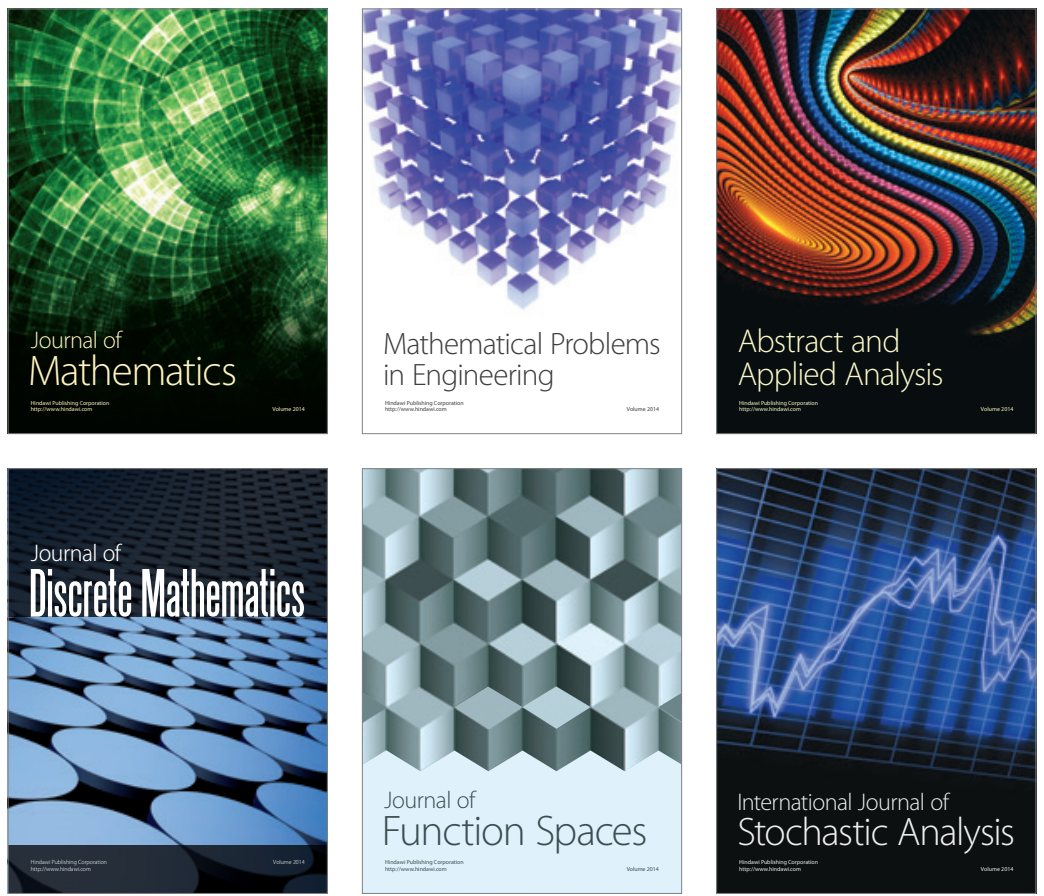

Journal of

Function Spaces

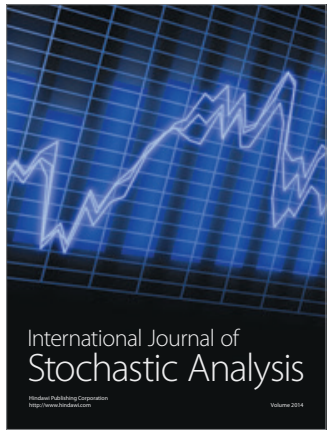

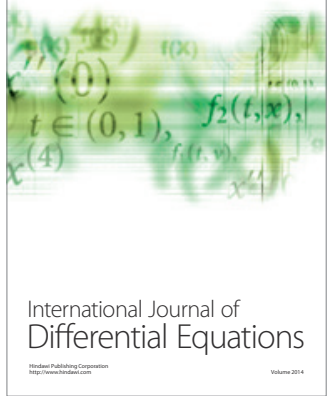
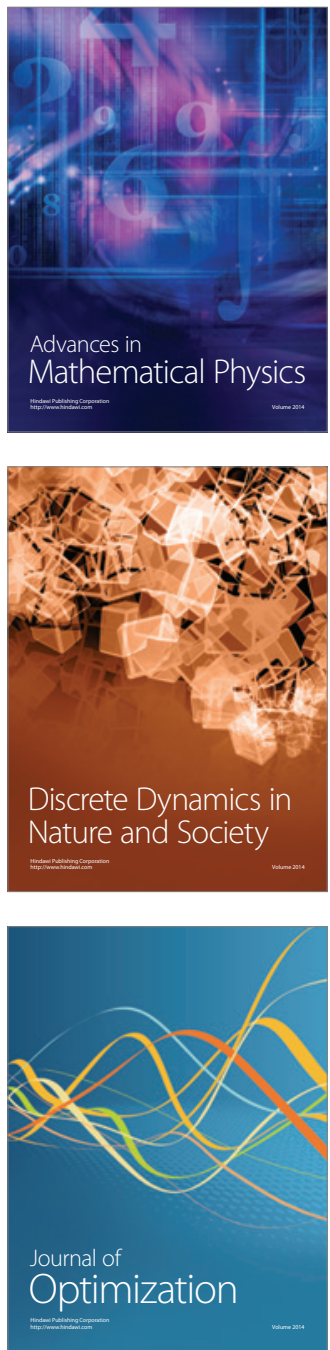